\title{
Anomalies, Fayet-Iliopoulos terms, and the consistency of orbifold field theories
}

\author{
R. Barbieri \\ Scuola Normale Superiore, Piazza dei Cavalieri 7, I-56126 Pisa, Italy \\ and INFN, Pisa, Italy \\ R. Contino and P. Creminelli \\ Scuola Normale Superiore, Piazza dei Cavalieri 7, I-56126 Pisa, Italy, \\ INFN, Pisa, Italy, \\ and Theory Division, CERN, CH-1211 Geneva 23, Switzerland \\ R. Rattazzi* and C. A. Scrucca \\ Theory Division, CERN, CH-1211 Geneva 23, Switzerland
}

(Received 8 April 2002; published 18 July 2002)

\begin{abstract}
We study the consistency of orbifold field theories and clarify to what extent the condition of having an anomaly-free spectrum of zero modes is sufficient to guarantee it. Preservation of gauge invariance at the quantum level is possible, although at the price, in general, of introducing operators that break the 5D local parity. These operators are, however, perfectly consistent with the orbifold projection. We also clarify the relation between localized Fayet-Iliopoulos (FI) terms and anomalies. These terms can be consistently added, breaking neither local supersymmetry nor gauge symmetry. In the framework of supergravity the localized FI term arises as the boundary completion of a bulk interaction term: given the bulk Lagrangian the FI is fixed by gauge invariance.
\end{abstract}

DOI: 10.1103/PhysRevD.66.024025

PACS number(s): 04.50.+h

\section{INTRODUCTION}

The existence of extra compact dimensions has been recognized since long ago to be quite a natural and appealing possibility, subject to rather mild constraints from presentday experiments. String theory, which is the only consistent candidate available at present for a microscopic and unified description of fundamental interactions, requires in fact at least six additional space dimensions, a fact that suggests that these might play some important role also from an effective field theory point of view [36].

Recently, renewed attention has been devoted to orbifold field theories with Scherk-Schwarz (SS) symmetry breaking [1]. Starting from five-dimensional (5D) supersymmetric theories, for instance, it has been possible to construct very simple and interesting extensions of the standard model with SS supersymmetry breaking [2,3], as well as grand-unified theories with SS gauge symmetry breaking [4]. Being nonrenormalizable, these models must be thought of as effective field theories valid up to some physical cutoff scale $\Lambda$. Incalculable UV effects can then be parametrized by writing the most general Lagrangian containing operators of arbitrarily high dimensions and with coefficients scaling with the suitable inverse powers of $\Lambda$. Working at energies $E \ll \Lambda$, and at finite order in an expansion in $E / \Lambda$ there are only a finite number of relevant operators. It is in this weaker sense that these theories are predictive. This is in complete analogy with the well-known case of pion interactions below the QCD scale. One relevant energy scale of these models is given by the compactification radius $E=1 / R$. Several inter-

\footnotetext{
*On leave from INFN, Pisa, Italy.
}

esting quantities of the low energy 4D theory can be written as a power series in $1 / R \Lambda$ and "weak" predictivity is satisfied only for $R \gg 1 / \Lambda$. Provided this condition holds, there are indeed some quantities which are nicely predicted in these models. These are the quantities for which there exists some $5 \mathrm{D}$ symmetry that forbids direct contributions by local 5D operators but is broken by the compactification. This ensures that the value of these quantities is controlled by calculable IR effects, and can thus be expressed in terms of other observables (see [3,5-7]). In particular the Higgs potential is protected by the original $N=2$ supersymmetry and falls in this category. Then electroweak symmetry breaking (ESB) occurs radiatively and is triggered by (global) supersymmetry breaking. Even more interestingly, very constrained models can be constructed with a single light Higgs scalar before ESB, such as the one proposed in [3], evading the need for at least two Higgs doublets in 4D supersymmetric extensions of the standard model. However, doubts were raised on the predictive power [8] and even on the consistency [9] of these models. The aim of this paper is to readdress these issues and discuss the conditions under which these models can be consistently defined.

In general, orbifold compactifications do not preserve the consistency of the higher-dimensional theory, because they correspond to a singular geometry. The consistency of orbifold models can however be studied from an effective field theory point of view, without invoking a more fundamental theory. In string orbifold models [10] (see [11,12] for specific examples with SS symmetry breaking), consistency is instead guaranteed as a consequence of more general principles, such as modular invariance. The twisted sectors arising at the fixed points provide blowing-up modes which allow to resolve the singularities through their vacuum ex- 
pectation value (VEV); orbifold compactifications can then be interpreted as singular limits of smooth manifold compactifications and this is sufficient to exclude any source of inconsistency. From a low energy supergravity point of view, anomalies cancel thanks to the presence of the twisted states and a generalization [13] of the Green-Schwarz (GS) mechanism [14] involving axions.

From an effective field theory viewpoint, one might think that a simple sufficient criterion to get a consistent orbifold theory could be to require an anomaly-free spectrum of chiral zero modes. This has been shown to hold true in the simplest case of $S^{1} / \mathbf{Z}_{2}$ orbifolds [15], but needs a qualification for $S^{1} /\left(\mathbf{Z}_{2} \times \mathbf{Z}_{2}^{\prime}\right)$ orbifolds, where anomalies may arise at fixed points and compensate each other only globally [9]. However, as already argued in [9], it is important to define the fate of the other symmetries in the theory in order to reach any sensible conclusion. The correct general statement turns out to be the following: under the assumption that the spectrum of massless modes is not anomalous, the theory can be made consistent and free of any localized anomaly, but in general at the price of introducing operators that are odd under a local 5D parity. The reason is that there is no regularization preserving simultaneously gauge invariance and the 5D local parity. Notice that these operators do not break the orbifold symmetry. As usual, the choices of preserving one or the other of the symmetries differ by the addition of a local counterterm that violates both symmetries; the localized anomalies arising in a regularization that forbids odd operators will be canceled thanks to an induced odd ChernSimons term in a gauge-invariant regularization. The connection between gauge anomalies and parity breaking by a Chern-Simons term is indeed a well-known property of field theories in odd dimensions.

In supersymmetric theories, another important issue concerns the generation of Fayet-Iliopoulos (FI) terms for the $U(1)$ gauge factors [16]. In 4D theories, this kind of term is not compatible with a supersymmetric and gauge-invariant vacuum state; it is radiatively generated only in the presence of $U(1)$-gravitational anomalies. Indeed, in supergravity a FI term is not gauge-invariant unless it is associated to an $R$ symmetry so that the gravitino has a charge $[17,18]$. In 5D orbifold models, we find that the situation is substantially different and two different kinds of FI terms can arise. The first type is truly associated with anomalies and cannot appear if the theory is consistently defined through a gaugeinvariant regularization. The second type corresponds to the boundary completion of a bulk term present in 5D supergravity. This bulk term is associated to, among other things, a kinetic mixing between the $U(1)$ photon and the graviphoton. The localized FI term is necessary to ensure full (supersymmetric) gauge invariance of the bulk term, and as such its value at the various boundaries is fixed.

The implication of our results for the single Higgs model of [3] is that the theory can be made fully consistent, but at the price of introducing odd operators. Divergent FI terms still arise at the fixed points, unless a specific regularization is assumed. For two Higgs models, the situation is different and one can consistently define these theories without the need to include odd operators.
The structure of the paper is the following. In Sec. II we discuss the consistency of odd operators in orbifold models and describe the local 5D parity that they violate. Our main discussion about anomalies is contained in Sec. III, while we leave for the Appendixes the explicit description of the regularizing procedures and an alternative approach based on Fujikawa's method. Section IV gives a detailed discussion about the consistency of FI terms at the orbifold fixed points and their relation with anomalies. Finally, in Sec. V we discuss the compatibility of our results with supergravity. Conclusions are drawn in Sec. VI.

\section{ODD OPERATORS ON ORBIFOLDS}

Consider a 5D model in which one of the spatial dimensions is compactified on a circle $S^{1}$, with coordinate $y$ $\in[0,2 \pi R]$. Consider also $5 \mathrm{D}$ parity $P_{5}$, corresponding to a reflection of the fifth coordinate around a fixed point, say $y$ $=0, y \rightarrow-y$, leaving the other coordinates unaffected. We can choose a basis where each field $\phi$ is either even or odd: $P_{5}[\phi(y)]=\eta_{\phi} \phi(-y)$, with $\eta_{\phi}= \pm 1$. One possible use of $P_{5}$ is to make it a (global) symmetry of the model by forbidding $P_{5}$-odd operators in the Lagrangian. Another, distinct possibility is to make it a gauge symmetry by projecting out of our original model all field configurations that do not satisfy the condition

$$
\phi(y)=P_{5}[\phi(y)]=\eta_{\phi} \phi(-y) .
$$

This projection corresponds to the construction of an $S^{1} / \mathbf{Z}_{2}$ orbifold theory. Notice that orbifolding corresponds to making the fields configuration $P_{5}$ invariant, without any reference to the Lagrangian. Indeed, after the projection, the addition of a parity-odd operator $\mathrm{O}^{-}$to the Lagrangian has no consequence because its integral over the fifth dimension trivially vanishes. Nevertheless, we can now add an odd operator, but with a coefficient that changes sign at the orbifold fixed points and is proportional to

$$
c(y)= \begin{cases}+1, & y \in(0, \pi R), \\ -1, & y \in(\pi R, 2 \pi R) .\end{cases}
$$

It is obvious that the introduction of parity-odd operators does not break the orbifold symmetry. Being a gauge symmetry the orbifold symmetry cannot be broken: non-invariant states are simply out of the theory. Indeed if we directly define the theory on the segment, with proper boundary conditions, we see that the idea of breaking the orbifold symmetry is nonsense. In the same way a nonzero profile for an odd field does not spontaneously break $P_{5}$, because of Eq. (1).

Having realized that parity-odd operators are allowed by the orbifolding, one may ask whether it is consistent to leave them out, or, in other words, if they can be forbidden by another symmetry. Always remaining in the simplest example of $S^{1} / \mathbf{Z}_{2}$, it is easy to see that the symmetry of reflection around the midpoint of the fundamental domain,

$$
\phi(y) \rightarrow \eta \phi(\pi R-y),
$$


forbids all parity-odd operators: with respect to Eq. (1), we have just changed the reflection point. Note that this symmetry exchanges the two boundaries: this gives no problem as all the fields have the same boundary conditions at 0 and $\pi R$. This discrete global symmetry is precisely the $\mathbf{Z}_{2}^{\prime}$ parity that will be gauged in $S^{1} /\left(\mathbf{Z}_{2} \times \mathbf{Z}_{2}^{\prime}\right)$ models. This symmetry is obviously explicitly broken by the introduction of odd terms and it is spontaneously broken by an odd profile $\left\langle\phi^{-}(y)\right\rangle$.

In the case of an $S^{1} /\left(\mathbf{Z}_{2} \times \mathbf{Z}_{2}^{\prime}\right)$ theory, the situation is slightly more involved. One may naively imagine to trivially extend the symmetry defined on $S^{1} / \mathbf{Z}_{2}$ : reflection around the midpoint $\pi R / 4$ of the fundamental domain. In this case we can choose one of the two possible parity assignments $\eta$ and $\eta^{\prime}$ given by the two orbifold actions:

$$
\phi(y) \rightarrow \eta \phi(\pi R / 2-y), \quad \phi(y) \rightarrow \eta^{\prime} \phi(\pi R / 2-y) .
$$

The point is that both symmetries defined in the above equation are broken by the boundary conditions: there are fields with different boundary conditions at 0 and $\pi R / 2$ and this is not compatible with the reflection that exchanges the two fixed points. The same may happen also on $S^{1} / \mathbf{Z}_{2}$ if we put fixed-point interactions that are different at 0 and $\pi R$, so that the two fixed points are physically distinguishable. The simple reason why on $S^{1} /\left(\mathbf{Z}_{2} \times \mathbf{Z}_{2}^{\prime}\right)$ we cannot find a global $5 \mathrm{D}$ parity symmetry is that we have already gauged the most general discrete symmetry group on $S^{1}$ [19]. However, even if it is impossible to define a global symmetry to avoid parity-odd operators, if we do not introduce odd operators from the beginning they will not be radiatively generated. This is because only the boundaries break the symmetries of Eq. (4), so that only nonlocal terms will be influenced. ${ }^{1}$ To put this idea on more solid ground we have to define this new symmetry locally. This is just a diffeomorphism that acts like one of the reflections (4) in an open set inside the fundamental domain and trivially outside. Obviously we need more than one chart to define this diffeomorphism over the full space. The boundaries break global parity very much in the same way as they break translations.

The presence of this local parity invariance ensures that odd terms will not be radiatively induced starting from a theory with only even terms. Note that, as this parity cannot be defined globally, it cannot be used to classify the physical states. Similarly changing the sign of the coefficients of all the odd operators in the Lagrangian leads to inequivalent theories. A relevant example, which we will encounter, showing this inequivalence, is a Dirac fermion mass.

Although the introduction of odd operators is fully consistent, something singular has to happen at the orbifold fixed points: either the coefficients of the odd operators have to change sign or the vacuum expectation value of an odd scalar has to jump. In the absence of this singular behavior the physics on the orbifold is the "smooth" projection of the

\footnotetext{
${ }^{1} \mathrm{We}$ are in a situation similar to a particle physics experiment held in a laboratory that is not parity symmetric: we do not expect that this will induce local interactions that violate parity.
}

physics on the full circle: in the presence of odd operators the relation with the original $S^{1}$ theory may be subtle, as is shown in the calculation of anomalies through index theorems in Appendix B.

\section{ANOMALIES}

In this section we want to study the simple case of 5D spinor electrodynamics compactified on an $S^{1} /\left(\mathbf{Z}_{2} \times \mathbf{Z}_{2}^{\prime}\right)$ orbifold. Before writing the Lagrangian and the fields, let us briefly establish our notation. We label our full space-time directions by Latin capital letters $M, N, \ldots=0,1,2,3,5$, where 5 indicates the compact one, and we indicate the noncompact directions by the Greek letters $\mu, \nu, \ldots=0,1,2,3$. We use the time-like Lorentz metric $\eta_{M N}=\operatorname{diag}(+,-,-$, $-,-)$. The 5D Dirac matrices $\Gamma^{M}$ are

$$
\begin{aligned}
& \Gamma^{\mu}=\left(\begin{array}{cc}
0 & \sigma_{+}^{\mu} \\
\sigma_{-}^{\mu} & 0
\end{array}\right) \equiv \gamma^{\mu}, \\
& \Gamma^{5}=-i\left(\begin{array}{cc}
-1 & 0 \\
0 & 1
\end{array}\right) \equiv-i \gamma^{5}, \quad \sigma_{ \pm}^{\mu}=\left(1, \pm \sigma^{i}\right),
\end{aligned}
$$

with $\sigma^{i}$ the Pauli matrices and $\gamma^{\mu}, \gamma^{5}$ the usual 4D matrices. We associate to the fields the labels $(++),(+-),(-,+)$ and $(-,-)$, depending on their parity under the $\mathbf{Z}_{2} \times \mathbf{Z}_{2}^{\prime}$ transformations. We are interested in the case where $A_{\mu}$ $=(+,+)$ and $A_{5}=(-,-)$ while the charged fermion satisfies $\psi(-y)=\gamma^{5} \psi(y)$ and $\psi(\pi R-y)=-\gamma^{5} \psi(y)$. Then if we write $\psi$ in terms of bispinors $\psi=\left(\chi, \bar{\chi}_{c}\right)$ we have $\chi$ $=(-,+)$ and $\chi_{c}=(+,-)$. The Lagrangian is

$$
\mathcal{L}=\int_{0}^{2 \pi R} d y\left[-\frac{1}{4 g^{2}} F^{M N} F_{M N}+\bar{\psi} i \Gamma^{M}\left(\partial_{M}-i A_{M}\right) \psi\right] .
$$

Even though we have not included a 5D mass term, no massless fermion survives the compactification because of the $\mathbf{Z}_{2} \times \mathbf{Z}_{2}^{\prime}$ parity assignments. The Kaluza-Klein decomposition of $\psi$ is

$$
\begin{aligned}
& \chi(x, y)=\sum_{n \geqslant 0} \xi_{n}^{-+}(y) \chi^{n}(x), \\
& \bar{\chi}_{c}(x, y)=\sum_{n \geqslant 0} \xi_{n}^{+-}(y) \bar{\chi}_{c}^{n}(x), \\
& \xi_{n}^{+-}(y)=a_{2 n+1} \cos \frac{2 n+1}{R} y, \\
& \xi_{n}^{-+}(y)=a_{2 n+1} \sin \frac{2 n+1}{R} y,
\end{aligned}
$$

where $a_{k}=1 / \sqrt{\pi R}$ for $k \neq 0$ and $a_{0}=1 / \sqrt{2 \pi R}$. These represent at each level $n$ a Dirac fermion with mass $M_{n}=(2 n$ $+1) / R$. The gauge field instead decomposes as 


$$
\begin{gathered}
A_{\mu}(x, y)=\sum_{n \geqslant 0} \zeta_{n}^{+}(y) A_{\mu}^{n}(x), \\
A_{5}(x, y)=\sum_{n \geqslant 1} \zeta_{n}^{-}(y) A_{5}^{n}(x), \\
\zeta_{n}^{+}(y)=a_{2 n} \cos \frac{2 n}{R} y, \\
\zeta_{n}^{-}(y)=a_{2 n} \sin \frac{2 n}{R} y,
\end{gathered}
$$

which represents at each level a massive vector with mass $2 n / R$. Notice that the orbifold projections act differently on the two chiralities so that this is a 4D chiral gauge theory. However 4D parity $P$ is still preserved combined with a 5D reflection about the midpoint $y=\pi R / 4$ of the fundamental domain: $y \rightarrow \pi R / 2-y$. The action of $P$ on the fields is

$$
\begin{gathered}
\chi\left(x_{0}, x_{i}, y\right) \rightarrow \bar{\chi}_{c}\left(x_{0},-x_{i}, \pi R / 2-y\right), \\
A_{\mu}\left(x_{0}, x_{i}, y\right) \rightarrow A^{\mu}\left(x_{0},-x_{i}, \pi R / 2-y\right), \\
\bar{\chi}_{c}\left(x_{0}, x_{i}, y\right) \rightarrow \chi\left(x_{0},-x_{i}, \pi R / 2-y\right), \\
A_{5}\left(x_{0}, x_{i}, y\right) \rightarrow-A_{5}\left(x_{0},-x_{i}, \pi R / 2-y\right) .
\end{gathered}
$$

This transformation leaves the boundary conditions unaffected, and thus $P$ survives after compactification. The KK gauge bosons $A_{\mu}^{n}$ have parity $(-)^{n+1}$, so that for $n$ even (odd) they couple through a vector (axial) current; also the scalars $A_{5}^{n}$ have parity $(-)^{n}$ and couple through a scalar (pseudoscalar) density for $n$ even (odd). In the same way it is easy to see that the system respects a charge conjugation $C$, defined as

$$
\begin{gathered}
\chi(y) \rightarrow \chi_{c}(\pi R / 2-y), \quad A_{\mu}(y) \rightarrow-A_{\mu}(\pi R / 2-y), \\
\chi_{c}(y) \rightarrow \chi(\pi R / 2-y), \quad A_{5}(y) \rightarrow A_{5}(\pi R / 2-y) .
\end{gathered}
$$

The KK modes transform under $C$ as $A_{\mu}^{n} \rightarrow(-)^{n+1} A_{\mu}^{n}$ and $A_{5}^{n} \rightarrow(-)^{n+1} A_{5}^{n}$. The use of these discrete symmetries will be useful in the following discussion on the anomaly.

It is known that on Minkowski spacetimes of odd dimensionality Abelian gauge theories are never anomalous (there are however global anomalies in the non-Abelian case $[20,21])$. However in the case at hand there are boundaries and one should be careful. In fact, as we already remarked, the system we are considering represents a chiral gauge theory from the 4D viewpoint: there are both vector $(V)$ and axial $(A)$ massive gauge fields. Very much as it happens in $4 \mathrm{D}$, there are in principle UV ambiguities in the definition of the three-point functions of the type $A V V$ and $A A A$. Moreover, as in 4D, one should not worry about $V V V$ and $A A V$, which vanish by charge conjugation. As noticed in Ref. [15] it is useful to arrange the fermions in an infinite vector of
Dirac spinors $\Psi=\left(\psi_{1}(x), \psi_{2}(x), \ldots\right), \quad \psi_{n}(x)$ $=\left(\chi^{n}(x), \bar{\chi}_{c}^{n}(x)\right)$. The gauge field is just an (infinite) matrix acting on this vector

$$
\mathcal{V}_{\mu}(x)+\gamma^{5} \mathcal{A}_{\mu}(x)=\int d y A_{\mu}(x, y)\left(Q_{V}(y)+\gamma^{5} Q_{A}(y)\right),
$$

where the charge matrices $Q_{V}$ and $Q_{A}$ are determined by the fermion wave functions

$$
\left(Q_{V, A}(y)\right)_{m n}=\frac{1}{2}\left[\xi_{m}^{+-}(y) \xi_{n}^{+-}(y) \pm \xi_{m}^{-+}(y) \xi_{n}^{-+}(y)\right] .
$$

Similarly we can write the coupling to $A_{5}$ via a scalar charge matrix

$$
\begin{aligned}
\mathcal{A}_{5}(x) & =\int d y A_{5}(x, y) \Omega(y), \\
\Omega(y) & =Q_{S}(y)+\gamma^{5} Q_{P}(y), \\
\left(Q_{S, P}(y)\right)_{m n} & =\frac{1}{2}\left[\xi_{m}^{-+}(y) \xi_{n}^{+-}(y) \mp \xi_{m}^{+-}(y) \xi_{n}^{-+}(y)\right],
\end{aligned}
$$

so that the fermion Lagrangian is

$$
\mathcal{L}=\bar{\Psi} \gamma^{\mu}\left(i \partial_{\mu}+\mathcal{V}_{\mu}(x)+\gamma^{5} \mathcal{A}_{\mu}(x)\right) \Psi-\bar{\Psi}\left(\mathcal{M}+i \mathcal{A}_{5}(x)\right) \Psi,
$$

where $\mathcal{M}$ is the mass matrix: $\mathcal{M}_{m n}=\delta_{m n} M_{n}$. The gauge transformations are represented by

$$
\begin{aligned}
\delta\left[\mathcal{V}_{\mu}(x)+\gamma^{5} \mathcal{A}_{\mu}(x)\right] & =\int d y \partial_{\mu} a(x, y)\left(Q_{V}(y)+\gamma^{5} Q_{A}(y)\right) \\
\delta \mathcal{A}_{5}(x) & =\int d y \partial_{y} a(x, y) \Omega(y) \\
\Psi(x) & \rightarrow \exp \left[i \int d y a ( x , y ) \left(Q_{V}(y)\right.\right. \\
& \left.\left.+\gamma^{5} Q_{A}(y)\right)\right] \Psi(x)
\end{aligned}
$$

under which invariance of the Lagrangian (16) follows thanks to the relation

$$
\partial_{y} \Omega(y)=\left[\mathcal{M}, Q_{V}(y)\right]+\gamma^{5}\left\{\mathcal{M}, Q_{A}(y)\right\},
$$

which is nothing but the statement that $\mathcal{M} \sim \gamma^{5} \partial_{5}$ on the fermion space. In the same way, the $5 \mathrm{D}$ divergence of the fermion current $\partial_{M} J^{M}$ at tree level is simply given by the variation of the Lagrangian (16) under a gauge rotation of the fermions, keeping the gauge fields unchanged

$$
\begin{aligned}
\partial_{M} J^{M}= & \partial_{\mu} \Psi \gamma^{\mu}\left(Q_{V}+\gamma^{5} Q_{A}\right) \Psi-i \Psi\left[\mathcal{M}, Q_{V}\right] \\
& +\gamma^{5}\left\{\mathcal{M}, Q_{A}\right\} \Psi .
\end{aligned}
$$


By the last equation, anomalies of the 5D current can be studied by using the known 4D results [15]. Indeed, Eq. (19) is simply a generalization of the familiar expression $\delta \mathcal{L}$ $=\partial_{\mu} J_{A}^{\mu}-2 i m \bar{\Psi} \gamma^{5} \psi$ encountered in the study of the chiral anomaly. By applying the known $4 \mathrm{D}$ results it is then straightforward to calculate the anomaly in the current conservation

$$
\begin{aligned}
\partial_{M} J^{M}(x, y)= & \frac{1}{96 \pi^{2}}\left[\operatorname{Tr}\left(Q_{L}(y) \mathcal{F}_{L}(x) \widetilde{\mathcal{F}}_{L}(x)\right)\right. \\
& \left.-\operatorname{Tr}\left(Q_{R}(y) \mathcal{F}_{R}(x) \widetilde{\mathcal{F}}_{R}(x)\right)\right],
\end{aligned}
$$

where $Q_{R, L}=Q_{V} \pm Q_{A}$ and $\mathcal{F}_{R, L}=\mathcal{F}_{V} \pm \mathcal{F}_{A}$. Using the completeness properties of the fermion eigenmodes, this expression can be written in a very simple and local form [15]. For the model at hand, it was calculated in Ref. [9], finding ${ }^{2}$

$$
\begin{aligned}
\partial_{M} J^{M}(x, y)= & \frac{1}{4}[\delta(y)-\delta(y-\pi R / 2)+\delta(y-\pi R) \\
& -\delta(y-3 \pi R / 2)] \frac{-1}{96 \pi^{2}} F_{\mu \nu} \widetilde{F}^{\mu \nu} .
\end{aligned}
$$

The form of this result, with "equal and opposite anomalies" at the two boundaries, could have easily been anticipated by simple arguments. First, the anomaly, if it exists, should be localized at the boundaries: inside the bulk, the UV properties of the triple current correlator are insensitive to the presence of the boundaries, then current conservation follows from the absence of anomalies in five dimensions. Second, in our case there is a $4 \mathrm{D}$ parity symmetry under which the two boundaries are exchanged, and for which the gauge parameter $a(x, y)$ is a scalar. Then, as $F \widetilde{F}$ is parity-odd, the contributions at the two boundaries should be equal and opposite.

The result (21) implicitly assumes the use of a mode-bymode regularization procedure that breaks gauge invariance explicitly. The same result can also be reproduced from a 5D point of view, for example by using a higher-derivative deformation of the fermionic kinetic term, as shown in Appendix A. Alternatively, one can also adopt Fujikawa's approach, in which the anomaly is associated to a Jacobian in the measure of the functional integral defining the effective action; see Appendix B.

\section{A. Chern-Simons term}

Let us keep arguing from a $4 \mathrm{D}$ perspective. It is well known that the 4D anomaly is determined up to the addition of local counterterms of the type $A_{\mu}^{m} A_{\nu}^{n} \partial_{\rho} A_{\sigma}^{r} \epsilon^{\mu \nu \rho \sigma}$. When at least two of the vectors are different, these counterterms can be used to shift the anomaly on just one of the three vertices, satisfying current conservation on the other two. This is what happens for the chiral anomaly, where the anomaly can be

\footnotetext{
${ }^{2}$ Actually our result is $1 / 3$ that of Ref. [9] as we must symmetrize with respect to the external vectors. See also Appendixes A and B.
}

shifted to the axial vector current preserving vector current conservation. Now, Eq. (20) corresponds to symmetrizing the anomaly with respect to the three (in general different) external legs and so, given a regulator, it represents a particular choice of local counterterms. In general, Eq. (20) should be considered up to the addition of such local counterterms. Indeed in our case there is yet another class of counterterms that can be added in order to shift (or eliminate) the anomaly. Of our big gauge group, only one $U(1)$ factor, associated to the zero mode $A_{\mu}^{0}$, corresponds to a linearly realized symmetry. The KK modes correspond to a gauged symmetry broken by Higgs mechanism for which $A_{5}^{n}$ are the (eaten) Goldstone bosons. Then one can also add terms of the type $A_{5}^{m} F_{\mu \nu}^{n} \widetilde{F}^{r} \mu \nu$, which can affect Eq. (21) since $A_{5}$ transforms inhomogeneously. In looking at this very special 4D system, one may then wonder whether the anomaly can be eliminated by a specific choice of these two classes of counterterms. Moreover, having the 5D picture dear, one may also ask whether a choice of counterterms exists which builds up into a local 5D operator. The answer to these questions is easy. Thinking directly in 5D, one immediately realizes that the addition of the Chern-Simons (CS) term

$$
\mathcal{L}_{C S}=\frac{1}{192 \pi^{2}} \int_{0}^{\pi R / 2} d y \epsilon^{M N O P Q} A_{M} F_{N O} F_{P Q}
$$

exactly cancels the anomaly of Eq. (21). It is easy to see that, when decomposed in KK modes, the CS term corresponds to local operators belonging to the two classes we mentioned above.

Note that in the above equation we have knowingly written the CS term by working on the single covering of the orbifold. When working on the full circle $S^{1}$, the CS would be

$$
\mathcal{L}_{C S}=\frac{1}{4} \frac{1}{192 \pi^{2}} \int_{S^{1}} d y \eta(y) \epsilon^{M N O P Q} A_{M} F_{N O} F_{P Q},
$$

where

$$
\eta(y)= \begin{cases}+1, & y \in(0, \pi R / 2) \cup(\pi R, 3 \pi R / 2), \\ -1, & y \in(\pi R / 2, \pi R) \cup(3 \pi R / 2,2 \pi R) .\end{cases}
$$

The almost obvious fact that such a CS term with a jumping coefficient could be used to cancel the anomaly was also noticed in Ref. [9], but not fully exploited. That was also because of some skepticism about the consistency of this parity-odd term with the orbifold projection. Indeed the CS term is odd, but only under the local 5D parity defined in Sec. II. As we explained there, the Lagrangian in Eq. (23) actually is perfectly consistent with the discrete symmetry that has been gauged to implement the orbifold projection. Of course, if we defined our field theory just by working on the segment and by assigning to fields either Dirichlet (+ fields) or Neumann ( - fields) conditions at each boundary, discontinuous parameters would not arise and there would be no orbifold symmetry to be confused about. Then there would manifestly be no issue. 
The whole discussion can be easily generalized to a nonAbelian gauge group. It is well known that non-Abelian global anomalies may exist, even in 5D Minkowski space, in a theory with an odd number of fermions [20]. In this case the action changes by $\pm \pi n$ under a homotopically nontrivial gauge transformation with winding number $n$; to restore gauge invariance a Chern-Simons term has to be added, violating in this way the 5D parity. On an orbifold, because of the presence of boundaries, the situation is quite different, as the anomaly is not a global effect but arises locally at the fixed points. For example, a theory with two fermions in 5D Minkowski space is not anomalous irrespectively of their gauge representation, while on the orbifold this is true only if the fermions are in conjugate representations.

\section{B. Gauge-invariant regularization}

We went through all of the above discussion of the anomaly because we wanted to better interpret the result of Ref. [9]. Instead we could have started by directly looking for a gauge-invariant regulator. Finding one would imply that there are no anomalies. In fact, this model can be regulated in the manner of Pauli and Villars (PV). First notice that, compatibly with gauge invariance and the orbifold boundary conditions, we can add a "jumping" fermion mass

$$
\mathcal{L}_{\text {mass }}=-\int_{S^{1}} d y m \eta(y) \bar{\psi} \psi \text {. }
$$

Similarly, we can then add a PV Dirac spinor $\Omega$ with wrong statistic and jumping mass $\eta(y) M$. Second we must study the PV spectrum when $|M| \rightarrow \infty$ to make sure that all the modes become infinitely heavy. We recall that this condition is not guaranteed in the presence of a jumping profile, as localized massless states can arise. For instance, on $S^{1} / \mathbf{Z}_{2}$ with one Dirac fermion, there is one massless chiral mode, which must survive the addition of a jumping mass $m c(y)$ for continuity reasons [22]. Since parity $P$ is a symmetry also in the presence of the mass term, the fermion KK modes will keep forming Dirac fermions. In order to study the spectrum, we need to consider only one chirality, say $\Omega_{L}$, which is a $(-+)$ field. The eigenvalue equation is

$$
\left(-\partial_{y}+M \eta(y)\right)\left(\partial_{y}+M \eta(y)\right) \xi_{n}^{-+}=M_{n}^{2} \xi_{n}^{-+}
$$

and the eigenvalues are given by the solutions of the equation

$$
-\frac{\lambda_{n}}{M}=\tan \frac{\lambda_{n} \pi R}{2}
$$

through the relation

$$
M_{n}^{2}=M^{2}+\lambda_{n}^{2}
$$

The behavior of the spectrum for $M \rightarrow \infty$ depends on the sign of $M$. For $M$ positive, all the solutions $\lambda_{n}$ of Eq. (28) are real, and then each $M_{n} \rightarrow \infty$ when $M \rightarrow+\infty$. For $M$ negative, and more precisely for $M<-2 /(\pi R)$, there is also an imaginary solution $\lambda_{0}=i \tilde{\lambda}$. As $M \rightarrow-\infty, \lambda_{0} \rightarrow i M$ and the correspond- ing eigenvalue $M_{0} \rightarrow 0$ (one has $M_{0} \sim \sqrt{2} M e^{-M \pi R / 2}$ ). This asymptotically massless mode is localized at $y=\pi R / 2$, while its right-handed partner is localized at the other boundary. This result is fairly intuitive. For $|M| \gg 1 / R$, positive or negative, the presence of localized light fermions at each boundary can be established by disregarding the effects of the other boundary. Only for $M$ negative are there localized zero modes compatible with the $\mathbf{Z}_{2}$ or $\mathbf{Z}_{2}^{\prime}$ boundary condition at each fixed point. A (set of) PV field(s) with positive mass is then our candidate regulator. As we will see in a moment, the selection of a sign for the regulator mass $M$ is associated to the sign of the CS term which cancels the anomaly.

We have explicitly checked that the fermion loop short distance singularities are regulated to an arbitrary degree by adding a combination of PV fields. Consider for instance the vector 3-point function. It is convenient to work in Euclidean momentum space along the compact directions and in position space for the fifth direction. We can for instance calculate the fermion propagator in the limit in which we take the second fixed point to infinity; for $y, y^{\prime}>0$ we have $(\tilde{p}$ $\left.\equiv \sqrt{p^{2}+m^{2}}\right)$ :

$$
\begin{aligned}
S\left(p ; y, y^{\prime}\right)= & \frac{1}{2 \tilde{p}}\left[\frac{e^{-\tilde{p}\left(y+y^{\prime}\right)}}{\tilde{p}+M}\left(\tilde{p} \gamma^{5}-p-M\right)\left(\tilde{p} \gamma^{5}+M\right)\right. \\
& +e^{-\tilde{p}\left|y-y^{\prime}\right|}\left(-\tilde{p} \gamma^{5} \cdot \operatorname{sgn}\left(y-y^{\prime}\right)\right. \\
& +\not p+M)],
\end{aligned}
$$

with obvious extension to $y, y^{\prime}<0$. In this mixed notation the 3-point vertex depends on both the three 4D external momenta $\left(p_{1}, p_{2}, p_{3}\right)$ and the three positions in the fifth dimension $\left(y_{1}, y_{2}, y_{3}\right)$, and of course there is a $d^{4} p$ integral over the virtual 4D momentum. UV ambiguities arise only when the $y_{i}$ coalesce to a point; otherwise, the loop momentum integral converges exponentially thanks to the propagator terms $\exp (-\tilde{p}|\Delta y|)$. However, for coinciding $y$ 's, the integrand is just an analytic function of the momenta and masses. So it can be made to vanish for $p \rightarrow \infty$ like $1 / p^{k}$ with arbitrary $k$ by the addition of a suitable number of PV fields with masses $M_{i}$ and charges $q_{i}$ satisfying relations of the type

$$
\sum_{i} q_{i}^{3}=1, \quad \sum_{i} q_{i}^{3} M_{i}^{n}=0 \quad \text { for } n>0
$$

here the first relation ensures that the leading divergence associated with the physical fermion (with charge $q=1$ ) is canceled. Then all the possible UV ambiguities in the gauge boson vertices can be eliminated in a manifestly gaugeinvariant way and we conclude that the theory is not anomalous. The presence of a gauge-invariant regulator allows us to power count the UV divergences by using gauge invariance. For 3-point vertices it is straightforward to see that the result must be finite: the only bulk operator that could, in principle, be logarithmically divergent is the CS term; however the CS cannot be made fully gauge invariant by the 

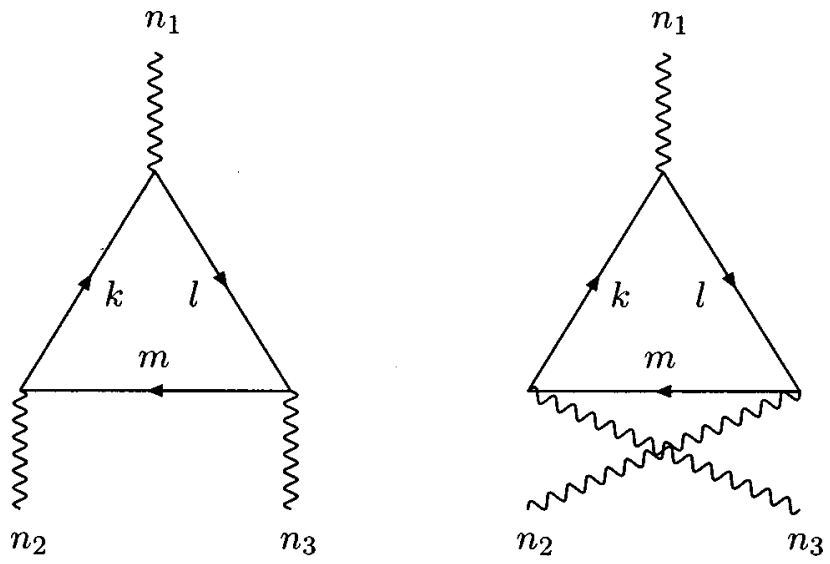

FIG. 1. The two diagrams contributing to the vector three-point function.

addition of local terms at the boundaries. Still, in order to properly define the 3-point vertex the addition of at least one PV field is necessary. In order to see this, it is easier to work with KK modes rather than with the mixed propagator. The basic story is the following. The only vertices that are potentially anomalous and need regulation are those for which the 3 vector wave numbers satisfy the relation $n_{1}+n_{2}+n_{3}$ = odd [9]. (These are the vertices of the type $A V V$ and $A A A$. The $V V V$ and $A A V$ vanish by charge conjugation, while those involving one external scalar are finite.) For these diagrams, there is only a finite number of choices of the internal fermion fifth momentum: there is no infinite $\mathrm{KK}$ sum and the situation is therefore exactly the same as in $4 \mathrm{D}$, with a linear divergence. This is essentially due to the fact that $n_{1}+n_{2}$ $+n_{3}=$ odd violates momentum conservation in the fifth direction. The presence of these diagrams is just a consequence of the boundary. Given the external bosons, there are two such diagrams (see Fig. 1). They are individually linearly divergent, but although their sum is finite the result is ambiguous since it depends on the routing of the integration momentum on each diagram. The addition of one PV regulator field resolves this ambiguity while preserving gauge invariance. Now for each diagram of Fig. 1 we subtract an (infinite) series of PV diagrams. After the subtraction the linear divergence is eliminated and there is no longer a momentum routing ambiguity. The series of PV diagrams works like a generalization of multi-PV regulators. What matters is that the leading divergence of the sum of PV matches exactly the leading divergence of the physical diagram. This is explained in detail in Appendix A.

We have argued that the anomaly can be eliminated either by adding a CS with jumping coefficient or by regulating the theory via a PV with jumping mass. What is the relation between the two approaches? The answer is pretty simple. Notice first of all that both the CS and the fermion mass are odd under the (local) 5D parity. Then, by symmetry and power counting, it is expected that for $M \rightarrow \infty$ there should remain a finite $\mathrm{CS}$ term. We can easily calculate its coefficient by looking at the triple vertex for three points inside the bulk. The term we are interested in corresponds to a single mass insertion and, for $M \rightarrow \infty$, it does not feel the presence of the boundaries, so that the result is the same one obtains in an infinite fifth dimension. By explicit calculation we find that the effective CS term is

$$
\mathcal{L}_{C S}^{e f f}=\frac{1}{4} \frac{1}{192 \pi^{2}} \frac{M}{|M|} \int_{S^{1}} d y \eta(y) \epsilon^{M N O P Q} A_{M} F_{N O} F_{P Q},
$$

which is precisely what we need to cancel the anomaly provided $M$ is positive. But $M$ positive is indeed the requirement imposed on our regulator by the decoupling requirement. Therefore the two pictures nicely match.

\section{THE FAYET-ILIOPOULOS TERM}

Given the connection between FI terms and mixed $U(1)$-gravitational anomalies in $4 \mathrm{D}$, it is natural to ask whether a similar relation persists in $5 \mathrm{D}$ orbifold theories as well.

It is well known that 4D theories with rigid supersymmetry admit a FI term for $U(1)$ vector multiplets: $\mathcal{L}_{F I}$ $=[\xi V]_{D}$. This term is manifestly supersymmetric. It is also invariant under the supergauge transformation $V \rightarrow V+\Lambda$ $+\bar{\Lambda}$, since $[\Lambda]_{D}=[\bar{\Lambda}]_{D}=0$. However, its presence destabilizes the vacuum and triggers the spontaneous breaking either of supersymmetry or of the $U(1)$ gauge symmetry (or of both).

In 4D supergravity theories, the situation is different: the naive extension of the rigid FI term is not gauge-invariant by itself. In the superconformal approach [23], with a compensator chiral multiplet $S_{0}$, a rigid FI term would be promoted to $\left[S_{0} \bar{S}_{0} \xi V\right]_{D}$, which is no longer invariant under $V \rightarrow V$ $+\Lambda+\bar{\Lambda}$ since $\left[S_{0} \bar{S}_{0} \Lambda\right]_{D}$ and $\left[S_{0} \bar{S}_{0} \bar{\Lambda}\right]_{D}$ are nonzero. To write a gauge-invariant generalization of a FI term, $S_{0}$ must transform under the $U(1)$ symmetry: a term of the form $\left[S_{0} \bar{S}_{0} e^{\xi V}\right]_{D}$ will be invariant, provided the compensator undergoes a super-Weyl transformation $S_{0} \rightarrow e^{-\xi \Lambda} S_{0}, \quad \bar{S}_{0}$ $\rightarrow e^{-\xi \bar{\Lambda}} \bar{S}_{0}[17,18]$. This implies that the $U(1)$ symmetry must in fact be a gauged version of the $U(1)_{R}$ symmetry: the FI term will induce a nonvanishing charge for the gravitino. In component fields we can check that the linear term $\left[S_{0} \bar{S}_{0} \xi V\right]_{D}=\xi e\left(D+(i / 4) \epsilon^{\mu \nu \rho \sigma} \bar{\psi}_{\mu} \gamma_{\nu} \psi_{\rho} A_{\sigma}+\cdots\right) \quad$ indeed contains a coupling of the $U(1)$ vector field $A_{\mu}$ to the gravitino $\psi_{\mu}$, beside the rigid FI term.

An alternative way to have a FI-like term in local supersymmetry is to introduce the superfield $V$ in combination with a chiral multiplet $\Phi$ transforming nonhomogeneously under the $U(1): \Phi \rightarrow \Phi-\Lambda$. One can then write a generic gauge-invariant Kähler potential of the form $K(\Phi+\bar{\Phi}+V)$; the linear piece in the expansion of $K$ will give rise to a FI term $K^{\prime}(\langle\Phi+\bar{\Phi}\rangle) V[13]$. In general, this mechanism gives, however, a mass term $\frac{1}{2} K^{\prime \prime}(\langle\Phi+\bar{\Phi}\rangle) V^{2}$ for the $U(1)$ gauge boson.

Summarizing, in four dimensions, a FI term is in general not gauge-invariant in the presence of gravity. If the theory is affected by mixed $U(1)$-gravitational anomalies this term is radiatively generated. More precisely, the anomaly and the FI term will be simultaneously generated at one loop, with a 
common coefficient given by the trace of the $U(1)$ charge over the spectrum of the model.

Given its connection with mixed anomalies in the 4D supergravity, we are interested in studying the possibility to have FI terms at the orbifold fixed points in the 5D theory. We will see that the situation is quite different with respect to the $4 \mathrm{D}$ case. Indeed, from the 4D point of view, the chiral multiplet $\Phi$ containing $A_{5}$ transforms inhomogeneously under gauge transformations and it has a Kähler potential of the form $K\left(\Phi+\bar{\Phi}-\partial_{5} V\right)$. As we shall see, this will allow for a consistent FI term, whose gauge variation is canceled by bulk terms. Furthermore, such a term will spontaneously break neither supersymmetry nor gauge symmetry, contrary to what happens in $4 \mathrm{D}$. Moreover we will see that the radiative generation of FI operators at the orbifold fixed points is not forbidden even in the absence of mixed anomalies.

\section{A. The $S^{1} / Z_{2}$ model}

We begin by considering the $S^{1} / \mathbf{Z}_{2}$ case, where one of the supersymmetries remains unbroken and one can efficiently use the superfield formalism with respect to this $N=1$ [24]. An $N=2$ vector multiplet decomposes into an $N=1$ vector multiplet $V=\left(A_{\mu}, \lambda_{1}, D\right)$ plus a chiral multiplet $\Phi$ $=\left((1 / \sqrt{2})\left(\Sigma+i A_{5}\right), \lambda_{2}, G\right)$, transforming in the adjoint representation of the gauge group. ${ }^{3}$ Similarly, an $N=2$ hypermultiplet decomposes into two chiral multiplets $H$ $=(\phi, \chi, F)$ and $H_{c}=\left(\phi_{c}, \chi_{c}, F_{c}\right)$. The kinetic Lagrangian of a hypermultiplet interacting with a $U(1)$ vector multiplet, in the presence of a FI term is

$$
\begin{aligned}
\mathcal{L}_{K}= & \frac{1}{g^{2}} \int d^{4} \theta\left[2 \xi(y) V+\left(\partial_{5} V-\frac{1}{\sqrt{2}}(\Phi+\bar{\Phi})\right)^{2}\right] \\
& +\frac{1}{4 g^{2}} \int\left[d^{2} \theta W^{\alpha} W_{\alpha}+\text { H.c. }\right]+\int d^{4} \theta\left[\bar{H} e^{q V} H\right. \\
& \left.+H_{c} e^{-q V} \bar{H}_{c}\right] \\
& -\int\left[d^{2} \theta H_{c}\left(\partial_{5}-\frac{q}{\sqrt{2}} \Phi-m(y)\right) H+\text { H.c. }\right],
\end{aligned}
$$

where $g$ is the gauge coupling and $q$ is a dimensionless charge. Notice the appearance of the covariant derivative in the extra dimension $\partial_{5}-(q / \sqrt{2}) \Phi[24,25]$. The above Lagrangian is manifestly supersymmetric and the orbifold projection implies $m(-y)=-m(y)$, and $\xi(-y)=\xi(y)$. Furthermore, $N=2$ bulk supersymmetry would imply that $m(y)$ and $\xi(y)$ are piecewise constant: $m(y)=m \cdot c(y)$ and $\xi(y)$ $=\xi_{0}$ plus contributions localized at the orbifold fixed points. For the bosonic components, we find (after eliminating the auxiliary fields $G, F$ and $F_{c}$ )

\footnotetext{
${ }^{3}$ In terms of $N=2$ auxiliary fields, one has $D=X_{3}-\partial_{5} \Sigma$ and $G$ $=X_{1}+i X_{2}[27]$.
}

$$
\begin{aligned}
\mathcal{L}_{K}= & \frac{1}{g^{2}}\left[-\frac{1}{4} F_{M N} F^{M N}+\frac{1}{2} \partial_{M} \Sigma \partial^{M} \Sigma\right. \\
& \left.+\frac{1}{2}\left(D+\partial_{5} \Sigma\right)^{2}-\xi(y) D\right]+\left|\mathcal{D}_{M} \phi\right|^{2}-\left[(m(y)+q \Sigma)^{2}\right. \\
& \left.+\partial_{5} m(y)+q\left(D+\partial_{5} \Sigma\right)\right]|\phi|^{2}+\left|\mathcal{D}_{M} \phi_{c}\right|^{2} \\
& -\left[(m(y)+q \Sigma)^{2}-\partial_{5} m(y)-q\left(D+\partial_{5} \Sigma\right)\right]\left|\phi_{c}\right|^{2} .
\end{aligned}
$$

Notice the appearance of the $\partial_{5} m$ mass terms. In the relevant case of a piecewise constant $m$, these contributions are localized at the boundary. Their presence ensures that the 5D supercurrent $S_{M}^{\alpha}$ is conserved everywhere, a necessary condition to define the supergravity version of the model. The equation of motion for $D$ is

$$
D=\xi(y)-\partial_{5} \Sigma+q g^{2}\left(|\phi|^{2}-\left|\phi_{c}\right|^{2}\right) .
$$

We see that differently from the 4D case, the neutral scalar $\Sigma$ appears in the equation of motion for $D$. This is due to the nonhomogeneous transformation law of the chiral multiplet $\Phi$ and allows us to find a supersymmetric $(D=0)$ and gauge-invariant vacuum state even in the presence of the FI term. In fact, the $D$-flatness condition can be solved with $\langle\phi\rangle=\left\langle\phi_{c}\right\rangle=0$ and $\partial_{5}\langle\Sigma(y)\rangle=\xi(y)$. For generic FI terms at the two fixed points it is in general impossible to satisfy the condition $D=0$ at both boundaries. An "integrability condition" $\int d y \xi(y)=0$ is necessary to have a supersymmetric vacuum state: we must have opposite FI terms at the two sides. This requirement is equivalent to having a vanishing FI term in the effective 4D action: this was expected, because, as we saw, a 4D FI term is not compatible with a gauge-invariant supersymmetric vacuum. If the integrability condition is satisfied, the scalar potential is

$$
\begin{aligned}
V_{\text {scalar }}= & {\left[(m(y)+q \Sigma)^{2}\left(|\phi|^{2}+\left|\phi_{c}\right|^{2}\right)\right.} \\
& \left.+\partial_{5}(m(y)+q \Sigma)\left(|\phi|^{2}-\left|\phi_{c}\right|^{2}\right)\right] .
\end{aligned}
$$

The net effect of the FI terms is then to shift the mass terms by the vacuum expectation value (VEV) of $\Sigma(y)$.

A FI term satisfying the integrability condition is in fact equivalent to shifting $\Phi$ by a stepwise constant. This can be easily seen from the Lagrangian (32): a nonvanishing $\langle\Phi$ $+\bar{\Phi}\rangle=\sqrt{2}\langle\Sigma(y)\rangle$ in the second term generates a term $-2\langle\Sigma(y)\rangle\left[\partial_{5} V-(1 / \sqrt{2})(\Phi+\bar{\Phi})\right]_{D}$, which, in rigid supersymmetry, is equivalent to a FI term with $\xi(y)=\partial_{5}\langle\Sigma(y)\rangle$ after integration by parts.

This relationship between the FI term and bulk operators turns out to be important to understand the supergravity extension. In particular it says that boundary FI terms are equivalent to the bulk operator $c(y)\left[\partial_{5} V-(1 / \sqrt{2})(\Phi\right.$ $+\bar{\Phi})]_{D}$ after integration by parts. In the rigid limit we are considering, the addition of $-(1 / \sqrt{2})(\Phi+\bar{\Phi})$ to the FI operator seems superfluous as it vanishes. However, it is important to keep it, as it shows that the FI is gauge-invariant independently of the flat supersymmetry background we are working on. Basically this FI term belongs to the same class 
as those arising in heterotic string models for anomalous $U(1)$ 's, where the dilaton plays the role played here by $\Phi{ }^{4}$ We therefore expect the gauge invariance of such a FI to be "robust" and to resist the extension to supergravity. Indeed, one can write a bulk operator of the form $c(y)\left[S_{0} \bar{S}_{0}\left(\partial_{5} V\right.\right.$ $-(1 / \sqrt{2})(\Phi+\bar{\Phi}))]_{D}=c(y) e\left(\partial_{5} D+(i / 4) \epsilon^{\mu \nu \rho \sigma} \bar{\psi}_{\mu} \gamma_{\nu} \psi_{\rho} F_{\sigma 5}\right.$ $+\cdots)$. In this way, the gravitino coupling arising in the supergravity completion of the rigid FI term becomes a nonminimal bulk coupling that is perfectly gauge-invariant.

We have seen that FI terms at the orbifold fixed points are quite different from the familiar FI in 4D. To complete the analysis we want to show that FI terms can be radiatively generated even in the absence of mixed anomalies, contrary to what happens in 4D. The calculation of the one-loop corrections to the FI term is most conveniently done working in Euclidean momentum space for the four noncompact directions and in configuration space for the fifth dimension, as in [6]. In this way many subtleties related to the sum over the KK tower disappear. It is easy to calculate ${ }^{5}$

$$
\xi(y)=q g^{2} \int \frac{d^{4} p}{(2 \pi)^{4}}\left(G^{+}-G^{-}\right)(p ; y, y),
$$

where $G^{+}$is the propagator for the even scalar $\phi$ and $G^{-}$ that for the odd scalar $\phi_{c}$, evaluated at points coinciding in the extra dimension. The propagator for the even field satisfies the equation

$$
\begin{aligned}
& {\left[p^{2}-\partial_{y}^{2}+m^{2}+2 m(\delta(y)-\delta(y-\pi R))\right] G^{+}\left(p ; y, y^{\prime}\right)} \\
& \quad=\frac{1}{2}\left(\delta\left(y-y^{\prime}\right)+\delta\left(y+y^{\prime}\right)\right),
\end{aligned}
$$

while the odd propagator satisfies the same equation without boundary mass terms, as it vanishes at the fixed points. The explicit solutions at coincident points in the fifth dimension are for $0 \leqslant y \leqslant \pi R\left(\tilde{p} \equiv \sqrt{p^{2}+m^{2}}\right)$ :

$$
\begin{aligned}
G^{+}(p ; y, y) & =\frac{\left[\cosh \tilde{p} y+\frac{m}{\tilde{p}} \sinh \tilde{p} y\right]\left[\cosh \tilde{p}(\pi R-y)-\frac{m}{\tilde{p}} \sinh \tilde{p}(\pi R-y)\right]}{2 \tilde{p}\left(1-\frac{m^{2}}{\tilde{p}^{2}}\right) \sinh \tilde{p} \pi R}, \\
G^{-}(p ; y, y) & =\frac{\sinh \tilde{p} y \sinh \tilde{p}(\pi R-y)}{2 \tilde{p} \sinh \tilde{p} \pi R}
\end{aligned}
$$

with obvious extension to $\pi R \leqslant y \leqslant 2 \pi R$.

The contributions of the two scalars tend to cancel in the bulk; in fact, we expect a divergent FI term to appear only at the boundaries. To study this divergent term, we can take the limit $R \rightarrow \infty$ in $G^{+}-G^{-}$, obtaining

$$
\left(G^{+}-G^{-}\right)(p ; y, y) \rightarrow \frac{1}{2}\left[\frac{e^{-2 \tilde{p} y}}{\tilde{p}+m}+\frac{e^{-2 \tilde{p}(\pi R-y)}}{\tilde{p}-m}\right] .
$$

We are left with a contribution for each boundary, exponentially suppressed in the bulk. Since a massive hypermultiplet on $S^{1} / \mathbf{Z}_{2}$ has zero modes for any value of its mass, it is not possible to regulate the theory in the manner of $\mathrm{PV}$; we therefore introduce a momentum cutoff $\Lambda$. The FI profile behaves as $\Lambda^{3}$ at distances shorter than $\Lambda^{-1}$. There are quadratic and linear divergences proportional to a $\delta$ function and

\footnotetext{
${ }^{4}$ This is completely obvious in the deconstructed version of the model [26], where the integrability condition corresponds to the requirement that the FI be purely along a nonlinearly realized $U(1)$, with zero overlap with the linearly realized one.

${ }^{5}$ No similar tadpole is generated for $\Sigma$; this would obviously be nonsupersymmetric.
}

a logarithmic divergence proportional to a $\delta^{\prime \prime}$; their coefficients can be extracted by using $\tilde{p} e^{-2 \tilde{p}|z|} \rightarrow \delta(z)$ $+\delta^{\prime \prime}(z) /\left(4 \tilde{p}^{2}\right)$ in the UV region $\tilde{p} \rightarrow \infty$ of the integral. One finds

$$
\begin{aligned}
\xi_{\mathrm{div}}(y)= & \frac{g^{2} q}{32 \pi^{2}}\left\{\Lambda^{2}[\delta(y)+\delta(y-\pi R)]\right. \\
& -2 m \Lambda[\delta(y)-\delta(y-\pi R)]+\frac{1}{2} \ln \frac{\Lambda}{|m|}\left[\delta^{\prime \prime}(y)\right. \\
& \left.\left.+\delta^{\prime \prime}(y-\pi R)\right]\right\}
\end{aligned}
$$

The last term does not correspond to a FI term in the usual sense, but is actually a higher-derivative operator.

Consider now the case of several hypermultiplets with charges $q_{i}$ and masses $m_{i}$. The total $U(1)$-gravitational anomaly is proportional to $\Sigma_{i} q_{i}$. If this anomaly vanishes, the first term in $\xi(y)$ vanishes too, as it must; otherwise, it would give a FI term in the $4 \mathrm{D}$ effective theory that is not gauge-invariant in the presence of gravity; the first term is a "standard" FI operator. One is then left with a residual FI 
term satisfying the integrability condition $\int d y \xi(y)=0$, which, as we have discussed above, corresponds to a gaugeinvariant bulk term, whose net effect is to correct the masses of the charged scalars to

$$
\delta m_{i}=-\frac{g^{2}}{32 \pi^{2}} q_{i} \sum_{j} q_{j} m_{j} \Lambda .
$$

Note that the radiatively induced FI operators correspond to an odd bulk operator which explicitly violates the $\mathbf{Z}_{2}^{\prime}$ global symmetry discussed in Sec. II. Indeed, this term is generated only by introducing mass terms that violate this symmetry in the tree-level Lagrangian.

\section{B. The $S^{1} /\left(\mathrm{Z}_{2} \times \mathrm{Z}_{2}^{\prime}\right)$ model}

Consider now the $S^{1} /\left(\mathbf{Z}_{2} \times \mathbf{Z}_{2}^{\prime}\right)$ orbifold. In this case no rigid supersymmetry is left unbroken globally: each boundary satisfies a different $N=1$ supersymmetry out of the $N$ $=2$. However the condition that the model be embeddable in $5 \mathrm{D}$ supergravity places strong constraints on the local Lagrangian. For instance, as in the previous section, a hypermultiplet bulk mass term should be completed by scalar masses localized at the boundaries. We would like to find the generalization of what we discussed in the $S^{1} / \mathbf{Z}_{2}$ case, where FI terms can be consistently put on the boundaries without inducing gauge or supersymmetry breaking. In this case we have to impose the $D$-flatness condition at each boundary, with respect to the residual $N=1$ supersymmetry of that fixed point: in this way locally we have no supersymmetry or gauge symmetry breaking; supersymmetry is broken only by nonlocal terms. The decomposition of the $N=2$ multiplets with respect to the two supersymmetries of the two boundaries gives for the auxiliary field of the vector multiplet $D$ $=X_{3}-\partial_{5} \Sigma$ and $D^{\prime}=X_{3}+\partial_{5} \Sigma$, where $X_{a}$ is the $S U(2)_{R}$ triplet of auxiliary fields of the $D=5$ vector multiplet [27]. The relative minus sign in the expressions for $D$ and $D^{\prime}$ can be understood as a consequence of the $R$-symmetry rotation of the triplet $X_{a}$, which is needed to go from an $N=1$ supersymmetry to the other. All the terms $D+\partial_{5} \Sigma$ in the Lagrangian (33) become $D-\partial_{5} \Sigma$ with respect to the other supersymmetry, and we can thus write explicitly the $D$-flatness conditions at the two boundaries:

$$
\begin{aligned}
D & =\xi_{1} \delta(y)-\partial_{5} \Sigma=0 \\
D^{\prime} & =\xi_{2} \delta(y-\pi R / 2)+\partial_{5} \Sigma=0 .
\end{aligned}
$$

We see that to solve both equations we must have equal (and not opposite as in $S^{1} / \mathbf{Z}_{2}$ ) FI terms on the boundaries. As in the $S^{1} / \mathbf{Z}_{2}$ case, we expect that such FI terms should be easily extended to supergravity: their gauge variation will be canceled by bulk terms, which explicitly break the local parity symmetry defined in Sec. II for the $S^{1} /\left(\mathbf{Z}_{2} \times \mathbf{Z}_{2}^{\prime}\right)$ case. When the FI terms at the two fixed points are different, (local) supersymmetry or gauge symmetry (or both) are spontaneously broken. In this case we expect the supergravity extension to be nontrivial and the gravitino charged under the $U(1)$.

Consider now the one-loop renormalization of the FI term induced by a charged hypermultiplet with scalars $\phi^{++}$and $\phi_{c}^{--}$, whose form is given by an equation analogous to Eq. (36). As in the $S^{1} / \mathbf{Z}_{2}$ case there will be mass terms on the fixed points. At each boundary one can use the superfield formalism, as in the previous section, to find the proper term that locally restores supersymmetry. It is easily found, by using the second term of Eq. (35) and the fact that we have to exchange $\phi$ and $\phi_{c}^{\dagger}$ to go from one rigid supersymmetry to the other, that in this case the two mass terms have the same sign at the two fixed points. Therefore we have a scalar potential of the form

$$
\begin{aligned}
V_{\text {scalar }}= & {\left[(m(y)+q \Sigma)^{2}\left(|\phi|^{2}+\left|\phi_{c}\right|^{2}\right)\right.} \\
& \left.+2 m \sum_{k=0}^{3} \delta(y-k \pi R / 2)|\phi|^{2}\right] .
\end{aligned}
$$

The propagator for the odd scalar is again unaffected by the boundary terms, whereas the even propagator satisfies

$$
\begin{gathered}
{\left[p^{2}-\partial_{y}^{2}+m^{2}+2 m \sum_{k=0}^{3} \delta(y-k \pi R / 2)\right] G^{++}\left(p ; y, y^{\prime}\right)} \\
\quad=\frac{1}{4} \sum_{k=0,1}\left(\delta\left(y-y^{\prime}+k \pi R\right)+\delta\left(y+y^{\prime}-k \pi R\right)\right) .
\end{gathered}
$$

Their explicit form for coincident points in the extra dimension is, for $0 \leqslant y \leqslant \pi R / 2$,

$$
\begin{aligned}
G^{++}(p ; y, y) & =\frac{\left[\cosh \tilde{p} y+\frac{m}{\tilde{p}} \sinh \tilde{p} y\right]\left[\cosh \tilde{p}\left(\frac{\pi R}{2}-y\right)+\frac{m}{\tilde{p}} \sinh \tilde{p}\left(\frac{\pi R}{2}-y\right)\right]}{4 \tilde{p}\left[\left(1+\frac{m^{2}}{\tilde{p}^{2}}\right) \sinh \tilde{p} \frac{\pi R}{2}+2 \frac{m}{\tilde{p}} \cosh \tilde{p} \frac{\pi R}{2}\right]}, \\
G^{--}(p ; y, y) & =\frac{\sinh \tilde{p} y \sinh \tilde{p}\left(\frac{\pi R}{2}-y\right)}{4 \tilde{p} \sinh \tilde{p} \frac{\pi R}{2}} .
\end{aligned}
$$


In the massless case, the result of [9] for the FI profile is easily recovered after integrating over the momentum. More generally, one can study the divergent contribution by taking as before the limit $R \rightarrow \infty$. The result is obviously the same as in the $S^{1} / \mathbf{Z}_{2}$ case because in this limit the propagator at one boundary is insensitive to the other boundary. The only change is the different sign of the mass at the second fixed point:

$$
\left(G^{++}-G^{--}\right)(p ; y, y) \rightarrow \frac{1}{4}\left[\frac{e^{-2 \tilde{p} y}}{\tilde{p}+m}+\frac{e^{-2 \tilde{p}(\pi R / 2-y)}}{\tilde{p}+m}\right] .
$$

The divergent part of the FI terms is then found to be

$$
\begin{aligned}
\xi_{\text {div }}(y)= & \frac{g^{2} q}{64 \pi^{2}}\left[\left(\Lambda^{2}-2 m \Lambda\right) \sum_{k=0}^{3} \delta(y-k \pi R / 2)\right. \\
& \left.+\frac{1}{2} \ln \frac{\Lambda}{|m|} \sum_{k=0}^{3} \delta^{\prime \prime}(y-k \pi R / 2)\right] .
\end{aligned}
$$

The terms proportional to the $\delta$ 's correspond to induced FI terms equal at the two boundaries. This, as we said, gives a vacuum that is both (locally) supersymmetric and gaugeinvariant. The $\delta^{\prime \prime}$ term gives instead a higher-derivative operator. Note that there is no logarithmic divergence in the $\delta$ terms, although one would naively expect one on powercounting grounds. The reason is that such a term would correspond to a bulk operator violating the local parity, with a coefficient proportional to $m|m|$; but such a term, being nonanalytic in the mass parameter, cannot be divergent.

Different from the $S^{1} / \mathbf{Z}_{2}$ case, a theory with a massless Higgs hypermultiplet of charge $q=1$ on $S^{1} /\left(\mathbf{Z}_{2} \times \mathbf{Z}_{2}^{\prime}\right)$ can be regularized with a set of PV fields, as already explained in Sec. III. In order to calculate the FI at one loop, the masses and charges of the regulators must satisfy $\Sigma_{i} q_{i}=1$ and $\Sigma_{i} q_{i} M_{i}=0$; this regularizes the quadratic and linear divergences in the $\delta$ terms, and also the logarithmic divergence in the $\delta^{\prime \prime}$ terms. In the limit $M_{i} \rightarrow+\infty$, we are left with a divergent contribution of the form

$$
\begin{aligned}
\xi_{\mathrm{div}}(y)= & \frac{g^{2}}{64 \pi^{2}}\left[-2 \sum_{i} q_{i} M_{i}\left|M_{i}\right| \sum_{k=0}^{3} \delta(y-k \pi R / 2)\right. \\
& \left.+\frac{1}{4} \sum_{i} q_{i} \ln \frac{\left|M_{i}\right|}{\mu} \sum_{k=0}^{3} \delta^{\prime \prime}(y-k \pi R / 2)\right],
\end{aligned}
$$

where $\mu$ is an arbitrary renormalization scale. In order to renormalize the theory, we have to add local counterterms that cancel $\xi_{\text {div }}$. After doing so, the one-loop correction to the FI term is a $U V$-finite distribution $\hat{\xi}(y)$ which, away from the boundaries, is purely determined by the 5D massless physical fields. Our introduction of a set of PV fields satisfying the two conditions $\Sigma_{i} q_{i}=1, \Sigma_{i} q_{i} M_{i}=0$ and of the local counterterms corresponds to making $\hat{\xi}(y)$ and $y^{2} \hat{\xi}(y)$ integrable and finite when $M_{i} \rightarrow \infty$. The integral $\int d y y^{2} \hat{\xi}(y)$ $\propto \log \mu R$ is infrared-divergent in the limit of infinite extra dimension: $R$ acts as an IR cutoff.

Had we chosen a set of PV fields satisfying $\Sigma_{i} q_{i} M_{i}^{2}=0$ (recall that we must take $M_{i}>0$ for the regulators to decouple), the FI terms would have been finite. We will see in Sec. V that this particular choice of regularization has a clear interpretation in the 5D supergravity extension of the theory. It is worth noting that, with this choice, the zero mode of $\hat{\xi}$ (i.e., its integral over the full circle) vanishes exactly, while we would naively have expected a nonzero answer scaling like $1 / R^{2}$. This result is less surprising when the calculation is done through a $\mathrm{KK}$ mode expansion. In that case, the zero mode of $\xi$, before regulation, is determined by just one diagram where the scalar zero mode circulates; it is therefore formally independent of $R$, although infinite. The regulated result then should be finite while being still $R$ independent, so it must be zero.

\section{SUPERGRAVITY EMBEDDING}

In models where the existence of a locally conserved supercurrent is crucial (as in [3]) to constrain the possible divergences appearing in the effective action, one is naturally led to consider a supergravity embedding. It is therefore important to show how what we said in the previous sections is explicitly extended to 5D supergravity, in particular the introduction of a gauge-invariant PV regulator with a stepwise mass term.

Every locally supersymmetric 5D theory must contain the gravity multiplet, composed by the graviton $g_{M N}$, a symplectic Majorana gravitino $\psi_{M}$ and a vector field $A_{M}$ : the graviphoton. If we add to the pure gravitational theory $n_{V}$ vector multiplets, the Lagrangian is completely determined by the geometry of the manifold $\mathcal{M}$ parametrized by the $n_{V}$ vector-multiplet scalars $\phi^{x}$. This geometry is specified starting from a $\left(n_{V}+1\right)$-dimensional space $\mathcal{C}$ with coordinates $b^{i}$ and Kähler potential $\mathcal{K}\left(b^{i}\right)=d_{i j k} b^{i} b^{j} b^{k}$. The scalar manifold $\mathcal{M} \subset \mathcal{C}$ is then defined through the constraint $\mathcal{K}\left(b^{i}\right)=6$ on which $b^{i}=b^{i}\left(\phi^{x}\right)$ and the metric $G_{i j}=-\frac{1}{2} \partial_{i} \partial_{j} \ln \mathcal{K}\left(b^{i}\right)$ on $\mathcal{C}$ induces a metric $G_{x y}=\partial_{x} b^{i} \partial_{y} b^{j} G_{i j}$ on $\mathcal{M}$. ${ }^{6}$ The Lagrangian is [29]

\footnotetext{
${ }^{6}$ The origin of this nice geometric description lies in the fact that 5D supergravities can be obtained as compactifications of elevendimensional supergravity on a Calabi-Yau space. The symmetric tensor $d_{i j k}$ appearing in the Kähler potential encodes the intersection numbers of the Calabi-Yau space and the constraint $\mathcal{K}=6$ corresponds to constant volume, whose fluctuations are described by an independent universal scalar field. See for example [28].
} 


$$
\begin{aligned}
\mathcal{L}= & -\frac{e}{2 \kappa^{2}}\left[R+G_{x y} \partial_{M} \phi^{x} \partial^{M} \phi^{y}+G_{i j} F_{M N}^{i} F^{j M N}\right. \\
& +\frac{d_{i j k}}{6 \sqrt{2}} \epsilon^{M N P Q R} A_{M}^{i} F_{N P}^{j} F_{Q R}^{k}-\frac{i}{2 \sqrt{2}}\left(\bar{\psi}_{M} \Gamma^{M N P Q} \psi_{N}\right. \\
& \left.\left.+2 \bar{\psi}^{P} \psi^{Q}\right) b_{i} F_{P Q}^{i}+\cdots\right],
\end{aligned}
$$

where the dots stand for terms involving the gauginos, which will not play any role in our discussion, and four-fermion terms. We indicate with $A^{i}$ the set of $n_{V}$ vectors and the graviphoton. Note that Chern-Simons terms are a fundamental ingredient of 5D supergravity.

Matter hypermultiplets can be easily introduced in the theory. Interactions with the vector multiplets can be achieved by gauging suitable isometries of the hypermultiplet scalar manifold (for a complete discussion see [30] and references therein ${ }^{7}$ ). A similar gauging is necessary also to generate mass terms and a potential for the hypermultiplets. In particular the hypermultiplet mass terms of rigid supersymmetry follow here from a gauging with the graviphoton: the mass of each hypermultiplet is proportional to its gravicharge. We are interested in a flat gauging, in which no cosmological constant is generated. It is well known that this is possible, provided that the gravitino has zero charge or, in other words, that the gauged isometry is not part of the $R$ symmetry.

Consider now such a supergravity theory on an orbifold. Under any orbifold symmetry, the bosonic fields $\left(g_{\mu \nu}, g_{55}, A_{5}\right)$ of the gravity multiplet must be taken even, while $\left(g_{\mu 5}, A_{\mu}\right)$ are odd. If we admit only even operators, the theory remains locally supersymmetric, both in the bulk and at the fixed points. Odd operators can be consistently introduced along the lines of [32]. To obtain a stepwise coefficient $g$, one promotes it to a field $G(x)$, invariant under supersymmetry: in this way the Lagrangian is no longer supersymmetric and its variation may be written as $\delta \mathcal{L}$ $=J_{M} \partial^{M} G$. This variation can be canceled by introducing a new four-form field $A_{4}$ with Lagrangian $\mathcal{L}_{\text {mult }}=A_{4} \wedge d G$ and transforming as $\delta A_{4}=-* J$, in such a way that $\delta(\mathcal{L}$ $\left.+\mathcal{L}_{\text {mult }}\right)=0$. The field $A_{4}$ acts as a Lagrange multiplier forcing $\partial_{M} G=0$ through its equation of motion, which yields back a constant $G$ on-shell, i.e. the situation we started with. At this stage, however, a nontrivial generalization can be obtained by introducing sources for $A_{4}$. Adding terms of the form $\mathcal{L}_{\text {source }}=\Sigma_{i} \mu_{i} \delta\left(y-y_{i}\right)\left(A_{4}+W\right)$, where $W$ is an additional term that is required to make $\delta \mathcal{L}_{\text {source }}=0$ on its own, the equation of motion of $A_{4}$ yields $\partial_{5} G=\Sigma_{i} \mu_{i} \delta\left(y-y_{i}\right)$, which implies that $G$ must have a piecewise constant profile on-shell. Notice that this extension is consistent provided the integrability condition $\Sigma_{i} \mu_{i}=0$ is satisfied. We have thus described a general method to introduce odd operators in supergravity, which, after the elimination of the Lagrange

\footnotetext{
${ }^{7}$ For a $D=4, N=2$ pregeometric approach, see [31].
}

multiplier 4-form, reduces to a proper addition of operators at the fixed points, which makes the full action invariant.

At this point, we are ready to discuss the generalization of the results obtained in the previous sections. From the above reasoning, it is clear that there is no obstruction to the introduction of a PV regulator with a jumping mass. The boundary mass terms found in Sec. IV arise here from the $W$ term, which is needed to make the fixed-point action invariant. This means that, even in supergravity, it is possible to regularize the theory in a gauge-invariant way, so that no anomaly will arise. As in the rigid limit, the PV regulator introduces a local CS term in the limit of infinite mass, with a jumping coefficient that is again described along the lines of [32]. Since the PV field has a nonvanishing gravicharge $r_{i}$ related to its mass, $M_{i} \propto r_{i} \kappa^{-1}$, a CS term with a photon and two graviphotons will also be generated, with a coefficient proportional to $\Sigma_{i} q_{i} r_{i}^{2}$.

In rigid supersymmetry we have seen that we can add FI terms on the boundaries with an arbitrary coefficient, modifying in this way the VEV of the scalar $\Sigma$. However, we also noticed that the FI should truly be regarded as an odd bulk operator, since in this way its full gauge invariance is manifest. We have also seen that in supergravity odd bulk operators are made consistent only by the addition of specific boundary terms [32]. It is then natural to expect that in supergravity the FI term is completely fixed by the bulk Lagrangian, i.e. by the structure constants $d_{i j k}$. Note that since the FI fixes the VEV profile of the gauge scalars $\Sigma$, then the structure constants $d_{i j k}$ themselves will determine where on the manifold $\mathcal{M}$ the vacuum is.

We will now explain this in more detail. In practice we will check that the additive renormalization of the hypermultiplet mass term, which is generated in the flat theory by the FI term, can also be calculated in the supergravity extension just by considering the $d_{i j k}$. Let us focus on a supergravity model with $n$ hypermultiplets and with a single vector field $B_{M}$ (which plays the role of hypercharge in the model of Ref. [3]) besides the graviphoton $A_{M}$. We consider the interesting situation where $B_{\mu}$ is even under the orbifold symmetry, while $A_{\mu}$ is necessarily odd. The two vectors gauge a $U(1)_{A} \times U(1)_{B}$ isometry of the hypermultiplet manifold, which we assume to be linearly realized. Around the $U(1)^{2}$ symmetric point, the action of the two isometries is just described in the linearized approximation by two commuting charge matrices $Q_{A}(y)=\eta(y) \hat{Q}_{A}$ and $Q_{B}$ acting on the $n$-dimensional hypermultiplet. Notice that since $A_{\mu}$ is odd its charge matrix undergoes an overall sign change at each fixed point [32]. Five-dimensional supergravity fixes the hypermultiplet mass matrix to be $M\left(Q_{A} b^{A}(\phi)+Q_{B} b^{B}(\phi)\right)$ where $M$ is just the 5D Planck scale up to some fixed constant (which is however unimportant in the following discussion). Notice that since the mass terms are odd operators, $b^{A}$ must be even and $b^{B}$ must be odd. Without loss of generality we can then choose $b^{B}=\phi / M$ where $\phi$ is an odd scalar, while $b^{A}$ is fixed in terms of $\phi$ through the constraint $d_{i j k} b^{i} b^{j} b^{k}$ $=6$. The scalar $\phi$ is the superpartner of $B$ when we take the flat limit. Now, let us start from a Lagrangian without parityodd operators in the pure gauge sector: only the constants 
$d_{A A A}=6$ and $d_{A B B}=1 /\left(M g_{B}^{2}\right)$ will be different from zero. Here we put in evidence the relation with the $U(1)_{B}$ coupling $g_{B}^{2}$. Since there are no odd terms in the gauge sector, following the procedure of Ref. [32] we should not add any boundary term to enforce local supersymmetry. In particular, there should be no boundary term involving just $\phi$, and since $\phi$ is odd it must vanish on the vacuum. Then the solution of the structural constraint will just be $b^{A}=1, b^{B}=0$, and the matter mass matrix will point along the graviphoton charge. What we have just outlined is the supergravity picture of a rigid theory without $U(1)_{B}$ FI term. The absence of a FI term is also consistent with the fact that the pure gauge supergravity Lagrangian is quadratic in $B: d_{A A B}=0$. Consider now a slight deformation of the previous model where we introduce a (small) $d_{A A B}=2 \epsilon \eta(y) d_{A B B}$. The new term introduces a kinetic mixing between $B$ and $A$ along with an $A A B$ CS term. Now we have terms that jump at the boundary and we must be careful to work out the boundary Lagrangian according to [32]. However, one can straightforwardly map the deformed theory back to the original one by noticing that through the field redefinition $A_{M}=\widetilde{A}_{M}, B_{M}$ $=\widetilde{B}_{M}-\epsilon \eta(y) \widetilde{A}_{M}$, the structure constants go back [up to $O\left(\epsilon^{2}\right)$ terms $]$ to the original ones, with $d_{A A B}=d_{B B B}=0$. [Notice that the field strengths rotate precisely as $A$ and $B$, without spurious terms, in spite of the presence of $\eta(y)$. This is thanks to $A_{M}$ being odd; a field redefinition of the type $A_{M} \rightarrow A_{M}+\eta(y) B_{M}$ would not be well behaved at the boundaries.] In the tilded basis we must have the same result as before: $\widetilde{b}^{A}=1, \widetilde{B}^{B}=0$, and by transforming back we find $b^{A} \simeq 1, b^{B}=-\epsilon \eta(y)$. The mass term of the deformed theory is therefore $M\left(Q_{A}-\epsilon \eta(y) Q_{B}\right)$ : a new term along $Q_{B}$ has been generated. The case we have just described should correspond to the supergravity extension of a flat theory with a FI $\propto d_{A A B}$. We have explicitly checked this by considering a theory where $d_{A A B}=0$ at tree level and by calculating the one-loop renormalizations of $A \wedge d A \wedge d B$ and of the $B$ FI, which are both $\propto \operatorname{Tr}\left(Q_{A} Q_{A} Q_{B}\right)$. We find that the correction to the matter mass matrix calculated with the above supergravity reasoning coincides with the direct calculation in rigid supersymmetry via the FI term. This explicitly shows that the FI term is just the flat limit remainder of a nonzero $d_{A A B}$.

An independent argument for this interpretation can be obtained by looking at the gravitino term $\epsilon^{\mu \nu \rho \sigma} \bar{\psi}_{\mu} \gamma_{\nu} \psi_{\rho} F_{\sigma 5}$ appearing in the $N=1$ supergravity completion of gaugeinvariant FI terms (see Sec. IV A), which in the $N=2$ theory must come from the last term in the Lagrangian (49). As we have seen, a vanishing FI term corresponds to only $b_{A}$ different from zero so that the gravitino couples only to the graviphoton. A nonvanishing FI term leads instead to $b_{B}$ $\neq 0$, implying a coupling between the gravitino and the corresponding field strength.

Summarizing, FI terms are seen as part of a parity-odd $N=2$ supergravity bulk operator, which contains a CS term with the corresponding gauge field and two graviphotons. If we cannot regulate the theory maintaining the local parity symmetry, these terms will be radiatively generated. The advantage of the supergravity point of view is that we can better understand the condition $\Sigma_{i} q_{i} M_{i}^{2}=0$ : the absence of FI terms is equivalent to a choice of regularization such that a CS term for two graviphotons and a photon is not generated or, equivalently, which does not mix the two vectors. We stress, however, that from the effective field theory viewpoint, there is no symmetry we can impose to avoid such operators, the only possible candidate, 5D parity, being broken to ensure gauge invariance.

\section{CONCLUSIONS}

We have shown that orbifold field theories with a nonanomalous spectrum of zero modes can in general be made gauge invariant only at the price of introducing odd operators. In particular there should effectively be a Chern-Simons term with a coefficient unambiguously fixed by the requirement of anomaly cancellation. These operators are allowed by the orbifold symmetry, but violate local 5D parity. The introduction of these new interactions has in general an important phenomenological impact, chiefly for nonsupersymmetric models. Indeed 5D parity plays the role of a discrete chiral symmetry; by its explicit breaking Dirac fermion masses can no longer be naturally forbidden. For instance, at the two-loop level, the CS term gives rise to a quartically divergent mass for charged fermions. In supersymmetric models such as [3] bulk supersymmetry limits the appearance of odd operators; mass terms for the hypermultiplets are however allowed: in particular a tree-level mass term could be added for the Higgs multiplet. If tree-level masses are set to zero, the nonrenormalization theorem guarantees that they can only be renormalized through the generation of FI terms $[8,33]$ and by finite, small, nonlocal corrections associated to the orbifold breaking of supersymmetry. Indeed the on-shell effect of a FI term in the limit of rigid supersymmetry is the same as a shift in the VEV of the scalar fields in the vector $N=2$ multiplets: it shifts the hypermultiplet masses. In the supergravity embedding a FI for a vector $B_{M}$ is associated to the presence of a CS term of the type $A \wedge d A \wedge d B$, where $A_{M}$ is the graviphoton. This fact suggests that the FI renormalization vanishes beyond one loop. This is because CS terms are in turn associated to anomalies and cannot be renormalized beyond one loop. Moreover, the one-loop renormalization of the FI term is given by the $B$ and graviphoton charges through a suggestively simple algebraic relation: $\xi \propto \operatorname{Tr}\left(Q_{A} Q_{A} Q_{B}\right) \propto d_{A A B}$. So while from pure symmetry considerations it is not possible to argue in favor of a vanishing FI term, nonetheless its form and renormalization properties are so restricted that it does not seem unreasonable to single out the case $\xi=0$ for phenomenological study. Of course a satisfactory explanation of $\xi=0$ can only come from a more fundamental theory. One possible direction is to try to obtain our 5D model as the low energy limit of 11D supergravity compactified on a Calabi-Yau space, then the $d_{i j k}$ and thus the FI are calculable topological quantities.

Note added. During the completion of the paper, Ref. [34] appeared, which has some overlap with our Sec. III. However, we disagree with the authors on their conclusion that the addition of odd operators spontaneously breaks the $\mathbf{Z}_{2}$ $\times \mathbf{Z}_{2}^{\prime}$ orbifold symmetry. 


\section{ACKNOWLEDGMENTS}

We are indebted to V. I. Zakharov for his helpful collaboration in the early stage of this work. We also thank G. Dall'Agata, J. P. Derendinger, S. Ferrara, A. Hebecker, M. Serone, L. Silvestrini and F. Zwirner for useful discussions. This work has been partially supported by the EC under TMR contract HPRN-CT-2000-00148.

\section{APPENDIX A: TWO DIFFERENT REGULATORS}

We give here the details of the computation of the anomaly exhibiting two different regulators for the threepoint function. The first one is a higher derivative deformation of the fermionic kinetic term, which breaks gauge invariance, preserves the local parity defined in Sec. III and reproduces the result of Eq. (21); the second one is the massive gauge invariant Pauli-Villars regulator, which leads to a nonanomalous theory with local parity explicitly broken. The Lagrangian for a 5D fermion on $S^{1} /\left(\mathbf{Z}_{2} \times \mathbf{Z}_{2}^{\prime}\right)$ with an arbitrary jumping mass term $M \eta(y)$ is

$$
\begin{aligned}
\mathcal{L}= & \int_{0}^{2 \pi R} d y\left[-\frac{1}{4 g^{2}} F^{M N} F_{M N}+\bar{\psi} i \Gamma^{M}\left(\partial_{M}-i A_{M}\right) \psi\right. \\
& -M \eta(y) \bar{\psi} \psi] .
\end{aligned}
$$

Using the decomposition of Eqs. (7) and (9), this gives

$$
\begin{aligned}
\mathcal{L}= & \sum_{k} \bar{\psi}_{k}\left(i \not b-M_{k}\right) \psi_{k}+\sum_{k, l, n} \alpha_{k l 2 n}^{+++}\left[P_{n}^{+} \bar{\psi}_{k} \gamma^{\mu} A_{\mu}^{2 n} \psi_{l}\right. \\
& \left.+P_{n}^{-} \bar{\psi}_{k} \gamma^{\mu} \gamma^{5} A_{\mu}^{2 n} \psi_{l}\right]+\sum_{k, l, n} \alpha_{k l 2 n}^{+--}\left[P_{n}^{+} \bar{\psi}_{k} i A_{5}^{2 n} \psi_{l}\right. \\
& \left.-P_{n}^{-} \bar{\psi}_{k} i \gamma^{5} A_{5}^{2 n} \psi_{l}\right],
\end{aligned}
$$

where $P_{n}^{ \pm}=\frac{1}{2}\left(1 \pm(-1)^{n}\right)$ and the overlaps $\alpha_{k l 2 n}^{ \pm \pm \pm}$are defined by

$$
\alpha_{k l 2 n}^{ \pm \pm \pm}=\int_{0}^{2 \pi R} d y \xi_{k}^{ \pm \mp}(y) \xi_{l}^{ \pm \mp}(y) \zeta_{2 n}^{ \pm}(y) \text {. }
$$

The divergence of the currents is found to be

$$
\begin{aligned}
\left(\partial_{\mu} J^{\mu}\right)^{2 n}= & \sum_{k, l} \alpha_{k l 2 n}^{+++}\left[P_{n}^{+} \partial_{\mu}\left(\bar{\psi}_{k} \gamma^{\mu} \psi_{l}\right)\right. \\
& \left.+P_{n}^{-} \partial_{\mu}\left(\bar{\psi}_{k} \gamma^{\mu} \gamma^{5} \psi_{l}\right)\right] \\
\left(\partial_{5} J^{5}\right)^{2 n}= & \sum_{k, l} i\left(M_{l}+(-1)^{n+1} M_{k}\right) \\
& \times \alpha_{k l 2 n}^{+++}\left[P_{n}^{+} \bar{\Psi}_{k} \psi_{l}-P_{n}^{-} \bar{\Psi}_{k} \gamma^{5} \psi_{l}\right] .
\end{aligned}
$$

The wave functions satisfy Eq. (26) and are given by

$$
\begin{aligned}
\xi_{k}^{+-}(y)= & \frac{1}{\sqrt{\pi R}}\left(1-\frac{\sin M_{k} \pi R}{M_{k} \pi R}\right)^{-1 / 2} \\
& \times \begin{cases}\sin M_{k}(y-\pi R / 2), & y \in[0, \pi R], \\
\sin M_{k}(3 \pi R / 2-y), & y \in[\pi R, 2 \pi R],\end{cases}
\end{aligned}
$$

$$
\xi_{k}^{-+}(y)=\xi_{k}^{+-}(y+\pi R / 2),
$$

the mass eigenvalues $M_{k}$ being defined by Eqs. (27) and (28). For each $k$ there is a $4 \mathrm{D}$ fermion $\psi_{k}$ with mass $M_{k}$ $=\sqrt{\lambda_{k}^{2}+M^{2}}$. In the limit $M \rightarrow 0$ we recover the massless wave functions of Eq. (8) and the usual spectrum, while for $M \rightarrow+\infty$ the whole tower of states becomes infinitely massive and decouples. When $M<-2 /(\pi R)$ one right-handed and one left-handed state appear localized respectively on $y$ $=0, \pi R / 2$ fixed points, becoming massless in the limit $M$ $\rightarrow-\infty$. This means that only a fermion with positive mass can play the role of a PV regulator. ${ }^{8}$

Now that we have shown the basic formulas, we introduce the first regulator, which consists of replacing the fermion kinetic term in Eq. (A1) with

$$
(i \not b-M \eta(y)) \rightarrow(i \not b-M \eta(y)) e^{\epsilon(i \not b+M \eta(y))(i \not b-M \eta(y))},
$$

where $\theta=\partial_{M} \Gamma^{M}$. The wave functions $\xi^{ \pm \mp}$ still satisfy Eq. (26) and the propagator of each KK mode $\psi_{k}$ gets an exponential convergence factor $\exp \epsilon\left(q^{2}-M_{k}^{2}\right)$ in momentum space. It is evident that this choice explicitly breaks 5D gauge invariance, even though it is even under the local parity defined in Sec. III. This procedure regulates both the 4D loop integral and the series over the KK modes in the threepoint function for a $5 \mathrm{D}$ fermion with (arbitrary) jumping mass. More in detail, working with the KK mode expansion, different diagrams can be formed when we consider the insertion of $\partial_{\mu} J^{\mu}, \partial_{5} J^{5}$ in the background of two gauge bosons. Depending on the external 5D momenta $n_{1}, n_{2}, n_{3}$ (see Fig. 1 ), any of the three vertices can be either vectorial, or axial, or scalar, or pseudoscalar. However, as previously stated, the sum of the two diagrams of Fig. 1 with an even number of $\gamma^{5}$ is odd under the charge conjugation of Eq. (12) and therefore vanishes. For the remaining diagrams, the external momenta are such that $n_{1}+n_{2}+n_{3}$ is odd, corresponding to an operator in the effective action which violates the conservation of momentum in the fifth direction and as such can only be a boundary operator. Dimensional analysis tells us that the naive divergence of these diagrams will be at most linear (and not quadratic), as expected for a boundary operator or, in other words, the sum over KK modes is convergent. This is clear in the massless limit where, if $n_{1}+n_{2}+n_{3}$ is odd, only

\footnotetext{
${ }^{8}$ What is actually relevant is the relative sign between the mass and $\Gamma^{5}$. Indeed, in five dimensions there are two inequivalent representations for $\Gamma^{5}$ which differ by a sign. Changing this convention also changes the sign of $M$ which gives localized states, but of course the physics remains the same.
} 
a finite number of KK modes circulate in the loop. It is clear from these considerations that there will be no contribution from the background $A_{5}$, as it vanishes on the boundary. The only remaining diagrams are of the type $A V V, A A A, V A V$ for $\left\langle\partial_{\mu} J^{\mu}\right\rangle$ and $P V V, P A A, S A V$ for $\left\langle\partial_{5} J^{5}\right\rangle$ (where the first label refers to the current).
Let us consider the graph $A V V$ with external 4D momenta $k_{1 \alpha}, k_{2 \beta}$, fixed modes $k, l, m$ running in the loop and 4D loop momentum $p$, as depicted in Fig. 1. Using the identity $\quad\left(k_{1}+k_{2}\right) \gamma^{5}=\left(\not p+/ k_{1}+M_{k}\right) \gamma^{5}-\left(\not p-k_{2}-M_{l}\right) \gamma^{5}$ $-\left(M_{k}+M_{l}\right) \gamma^{5}$, the trace over Dirac matrices can be split into three terms

$$
\begin{aligned}
& \operatorname{Tr}\left[\left(k_{1}+k_{2}\right) \gamma^{5} \frac{1}{p+k_{1}-M_{k}} \gamma^{\alpha} \frac{1}{p-M_{m}} \gamma^{\beta} \frac{1}{\not p-k_{2}-M_{l}}\right]+\text { symm. } \\
&=-\left(M_{k}+M_{l}\right)\left\{\operatorname{Tr}\left[\gamma^{5} \frac{1}{p+k_{1}-M_{k}} \gamma^{\alpha} \frac{1}{p-M_{m}} \gamma^{\beta} \frac{1}{p-k_{2}-M_{l}}\right]+\text { symm. }\right\} \\
&-\left\{\operatorname{Tr}\left[\gamma^{5} \gamma^{\alpha} \frac{1}{p-M_{m}} \gamma^{\beta} \frac{1}{p-k_{2}-M_{l}}\right]+\text { symm. }\right\}-\left\{\operatorname{Tr}\left[\gamma^{5} \frac{1}{p+k_{1}-M_{k}} \gamma^{\alpha} \frac{1}{p-M_{m}} \gamma^{\beta}\right]+\text { symm. }\right\} .
\end{aligned}
$$

The first term converges when integrated over $p$, even if one removes the regulator, and gives a contribution exactly equal and opposite to the $P V V$ insertion of $\partial_{5} J^{5}$. The other two, instead, would diverge linearly without the regulator, even if their sum is convergent. The presence of the regulator is then crucial to define each term separately by removing the routing ambiguity. More explicitly, using the regularization recipe of Eq. (A6), the sum of the last two terms in Eq. (A7) gives an anomalous piece $\sim \epsilon^{\alpha \mu \beta \nu} k_{1 \mu} k_{2 \nu}$ which does not depend on the mass of the modes, in the same way as happens in 4D. Similar considerations apply for the other diagrams $A A A, V A V$ and $P A A, S A V$ and resumming over all KK modes one finds the result of Eq. (21). We stress that the anomaly we found does not depend on the value of the mass ${ }^{9}$ $M$ and this is an indication that a massive fermion could act as a regulator itself.

Let us now discuss the Pauli-Villars regularization. In order to be a regulator for the theory with a massless fermion, the massive PV must resolve the ambiguity coming with the last two terms in Eq. (A7). This is exactly what happens. An arbitrary shift of the dummy variable in a linearly divergent integral corresponds to a surface finite contribution; in our case, a shift of the loop momentum $p \rightarrow p+a$ in the integral of the last two terms in Eq. (A7) gives a finite term that does not depend on the KK masses:

$$
\begin{aligned}
\Delta^{\alpha \beta}(a) & =2 i \pi^{2} \frac{a^{\mu}}{(2 \pi)^{4}} \lim _{p \rightarrow \infty} p^{2} p_{\mu} \frac{\operatorname{Tr}\left(\gamma^{5} \gamma^{\alpha} p \gamma^{\beta}\left(k_{1}+k_{2}\right)\right)}{p^{4}} \\
& =\frac{1}{8 \pi^{2}} \epsilon^{\alpha \mu \beta \nu} a_{\mu}\left(k_{1}+k_{2}\right)_{\nu} .
\end{aligned}
$$

\footnotetext{
${ }^{9}$ This extends the result of Ref. [9] and shows that what is found in [15] holds true also for the $S_{1} / \mathbf{Z}_{2} \times \mathbf{Z}_{2}^{\prime}$ orbifold.
}

Summing over all KK modes, one gets an arbitrary piece in the $A V V$ amplitude proportional to the sum of the overlap integrals

$$
I_{n_{1} n_{2} n_{3}}=\sum_{k, l, m} \alpha_{k l 2 n_{1}}^{+++} \alpha_{k m 2 n_{2}}^{+++} \alpha_{l m 2 n_{3}}^{++++}
$$

Using the completeness relations for our basis of wave functions, without ever using their expression, it is not difficult to show that $I_{n_{1} n_{2} n_{3}}$ does not depend on the mass $M$ but only on the external modes; more precisely ${ }^{10}$

$$
I_{n_{1} n_{2} n_{3}}=\frac{a_{2 n_{1}} a_{2 n_{2}} a_{2 n_{3}}}{2} \text { for } n_{1}+n_{2}+n_{3} \text { odd. }
$$

From this relation, it follows that the shift in the amplitude cancels when the PV contribution is added to the physical (massless) one or, in other words, that the leading divergence of the sum of the PV modes matches exactly the leading divergence of the physical diagram. We conclude that a massive $5 \mathrm{D}$ fermion with a positive jumping mass regulates the massless theory, removing any ambiguity.

We have shown that the regulator (A6) breaks the 5D gauge invariance, and this causes an anomalous term to appear. The 5D PV, on the contrary, preserves the gauge symmetry and the sum of the last two terms in Eq. (A7), regulated by the corresponding PV contribution, now vanishes. Again, the first term in Eq. (A7) is canceled by the $P V V$ insertion of $\partial_{5} J^{5}$, analogously to what we found with the regulator (A6). The same happens with $A A A, P A A$, and $V A V, S A V$, so that $\partial_{M} J^{M}=0$ and the theory is nonanomalous.

\footnotetext{
${ }^{10}$ When the external modes satisfy momentum conservation, $n_{1}$ $+n_{2}=n_{3}$, then $I_{n_{1} n_{2} n_{3}}$ diverges and indeed in this case an infinite number of KK modes circulate in the loop.
} 


\section{APPENDIX B: FUJIKAWA'S APPROACH AND INDEX THEOREMS}

In this appendix we will show how one can compute anomalies in a 5D orbifold theory using Fujikawa's method [35], in which anomalies are interpreted as the variation of the measure for fermions in the functional integral for the effective action, making the arguments outlined in [9] more precise.

We consider the $U(1)$ gauge transformations of a $5 \mathrm{D}$ Dirac spinor $\psi$ :

$$
\psi \rightarrow e^{i \alpha(x)} \psi, \quad \bar{\psi} \rightarrow e^{-i \alpha(x)} \bar{\psi}
$$

The variation of the measure under an infinitesimal transformation of this type is

$$
\begin{aligned}
& \mathcal{D} \psi \mathcal{D} \bar{\psi} \rightarrow \exp \left[-i \int d^{5} x \alpha(x)\left(\operatorname{Tr}_{\left\{\psi_{n}\right\}} 1-\operatorname{Tr}_{\left\{\bar{\psi}_{n}\right\}} \mathbb{1}\right)\right] \\
& \times \mathcal{D} \psi \mathcal{D} \bar{\psi},
\end{aligned}
$$

where $\left\{\psi_{n}(x)\right\}$ and $\left\{\bar{\psi}_{n}(x)\right\}$ are the eigenfunctions of $D^{\dagger} D$ and $D D^{\dagger}$, where $D$ is the Dirac operator. On the circle, $D$ $=D^{\dagger},{ }^{11}$ contrarily to what happens for a 4D Weyl spinor and therefore the anomaly trivially vanishes.

Consider now the case of an orbifold projection. The traces in Eq. (B2) must then be restricted to those states left invariant by the projection, by inserting the proper projection operator. Whenever the projection acts differently on $\psi$ and $\bar{\psi}$, the anomaly will no longer vanish. In the simplest case of $S^{1} / \mathbf{Z}_{2}$ generated by a reflection $R: y \rightarrow-y$ acting chirally on the fermions,

$$
\psi(-y)=\gamma^{5} \psi(y), \quad \bar{\psi}(-y)=-\bar{\psi}(y) \gamma^{5}
$$

Eq. (B2) becomes

$$
\begin{aligned}
\mathcal{D} \psi \mathcal{D} \bar{\psi} \rightarrow & \exp \left[-i \int d^{5} x \alpha(x)\left(\operatorname{Tr}_{\left\{\psi_{n}\right\}} \frac{1+Q}{2}\right.\right. \\
& \left.\left.-\operatorname{Tr}_{\left\{\bar{\psi}_{n}\right\}} \frac{1-Q}{2}\right)\right] \mathcal{D} \psi \mathcal{D} \bar{\psi},
\end{aligned}
$$

where $Q=R \gamma^{5}$. Since the two sets $\left\{\psi_{n}\right\}$ and $\left\{\bar{\psi}_{n}\right\}$ are equivalent, one finally finds an anomaly given by

$$
\mathcal{L}(x) \rightarrow \mathcal{L}(x)+\alpha(x) A(x) ; \quad A(x)=-\operatorname{Tr}_{\left\{\psi_{n}\right\}} Q
$$

As in the standard case, it is possible to give an index interpretation of this expression. The key ingredient to do so is that $Q$ satisfies $\{D, Q\}=0$ and $Q^{2}=1$. Since $\left[D^{2}, Q\right]=0$, the two operators can be simultaneously diagonalized and one can show that only zero modes of $D^{2}$ contribute to $\operatorname{Tr} Q$. In fact, for any nonzero mode $\psi_{n}$ of $D^{2}$, there exists another

\footnotetext{
${ }^{11}$ We are implicitly assuming an analytic continuation to Euclidean space, to have a Hermitian Dirac operator.
}

mode, $D \psi_{n}$, with the same $D^{2}$ eigenvalue but opposite $Q$. Therefore we can rewrite the integral of Eq. (B5) as

$$
\int d^{5} x A(x)=-\left(n_{Q=+}^{D^{2}=0}-n_{Q=-}^{D^{2}=0}\right) .
$$

We can write this expression as an explicit index because if we go to the basis that diagonalizes $Q$ we have

$$
\begin{aligned}
Q & =\left(\begin{array}{cc}
1 & 0 \\
0 & -1
\end{array}\right), D=\left(\begin{array}{cc}
0 & D_{+}^{\dagger} \\
D_{+} & 0
\end{array}\right), \\
D^{2} & =\left(\begin{array}{cc}
D_{+}^{\dagger} D_{+} & 0 \\
0 & D_{+} D_{+}^{\dagger}
\end{array}\right),
\end{aligned}
$$

so that Eq. (B6) can be rewritten as a function of $\operatorname{Ker} D_{+}$:

$$
\begin{aligned}
\int d^{5} x A(x) & =-\left(\operatorname{dim} \operatorname{Ker} D_{+}-\operatorname{dim} \operatorname{Ker} D_{+}^{\dagger}\right) \\
& =-\operatorname{index} D_{+} .
\end{aligned}
$$

We can go further and express the integrated anomaly in terms of the gauge connection, as in the usual 4D case. To do so it is useful to relate the trace of Eq. (B5) to the trace over $\gamma^{5}$ restricted to the fixed points of $R$ :

$$
\operatorname{Tr}_{\left\{\psi_{n}\right\}} Q=\frac{1}{2}[\delta(y)+\delta(y-\pi R)] \operatorname{Tr}_{\left\{\psi_{n}\right\}} \gamma^{5}
$$

To obtain this relation, we regularize the trace in an explicitly gauge-invariant way through the 5D Dirac operator:

$$
\begin{aligned}
A(x)= & -\sum_{k} \psi_{k}^{\dagger}(x, y) Q \psi_{k}(x, y) \\
= & -\sum_{k} \psi_{k}^{\dagger}(x, y) \gamma^{5} \psi_{k}(x,-y) \\
= & -\lim _{M \rightarrow \infty y \rightarrow x} \lim _{\operatorname{Tr}} \gamma^{5} \exp \left[-(D / M)^{2}\right] \delta(x-y) \\
& \times[\delta(2 y)+\delta(2 y-2 \pi R)] .
\end{aligned}
$$

Note that with respect to the standard $4 \mathrm{D}$ case we have an additional piece in the Dirac operator: $\Gamma^{5}\left(\partial_{5}-i A_{5}\right)$. Actually this extra piece does not contribute because the trace is nonzero only when we take $\gamma^{5}$ with four 4D gamma matrices coming from the expansion of the exponential. We thus obtain

$$
A(x)=\frac{1}{2}[\delta(y)+\delta(y-\pi R)] \frac{-1}{32 \pi^{2}} F_{\mu \nu} \widetilde{F}^{\mu \nu} .
$$

Note that this result differs by a factor of 3 from what we obtained in the evaluation of triangle diagrams. This is a consequence of the fact that we have regulated the trace (B10) in a way that explicitly preserves gauge invariance with respect to the background fields. This corresponds to an asymmetric treatment of the three legs of the triangle dia- 
gram. To restore the symmetry one would have to add local counterterms, obtaining in this way:

$$
A(x)=\frac{1}{2}[\delta(y)+\delta(y-\pi R)] \frac{-1}{96 \pi^{2}} F_{\mu \nu} \widetilde{F}^{\mu \nu} .
$$

We have recovered with Fujikawa's approach the gauge anomaly on $S^{1} / \mathbf{Z}_{2}$, originally calculated in [15] without symmetrization [i.e. they obtained Eq. (B11)]. The integrated anomaly is that of a Weyl fermion: the orbifold projection leaves in fact one unpaired massless state.

The whole discussion can be applied also to the $S^{1} /\left(\mathbf{Z}_{2}\right.$ $\times \mathbf{Z}_{2}^{\prime}$ ) case. Now we have to project as in Eq. (B4) with respect to both the $\mathbf{Z}_{2}$ 's and the relevant trace turns out to be

$$
A(x)=-\frac{1}{2} \operatorname{Tr}_{\left\{\psi_{n}\right\}}\left(\gamma^{5} R+\gamma^{5} R^{\prime}\right) .
$$

The calculation follows precisely the previous one, giving the result (21) as obtained in [9], apart from the already discussed factor $1 / 3$.
All the conclusions drawn in the previous sections can be reobtained in this approach. In particular, note that Fujikawa's calculation does not tell us anything about the possibility of canceling the anomalous variation of the fermion measure by local counterterms. As we discussed, in the $S^{1} /\left(\mathbf{Z}_{2}\right.$ $\times \mathbf{Z}_{2}^{\prime}$ ) case the addition of a heavy fermion with a stepwise mass gives a contribution to the calculation of the anomaly that cancels the contribution of the light states. This can be checked in this context: the anomaly here appears in the measure of integration, so that it does not change if we add a mass for the fermion. ${ }^{12}$ Usually this does not help to cancel anomalies because no fermion that is allowed by a given symmetry to have a mass can contribute to the anomaly for that symmetry; here this is not the case.

\footnotetext{
${ }^{12}$ In particular it tells us that the anomaly does not depend on the mass profile $m(y)$, as it was explicitly checked in Ref. [15] for the $S^{1} / \mathbf{Z}_{2}$ case.
}

[1] J. Scherk and J.H. Schwarz, Phys. Lett. 82B, 60 (1979); Nucl. Phys. B153, 61 (1979).

[2] A. Pomarol and M. Quiros, Phys. Lett. B 438, 255 (1998); A. Delgado, A. Pomarol, and M. Quiros, Phys. Rev. D 60, 095008 (1999).

[3] R. Barbieri, L.J. Hall, and Y. Nomura, Phys. Rev. D 63, 105007 (2001).

[4] Y. Kawamura, Prog. Theor. Phys. 103, 613 (2000); 105, 999 (2001); G. Altarelli and F. Feruglio, Phys. Lett. B 511, 257 (2001); L.J. Hall and Y. Nomura, Phys. Rev. D 64, 055003 (2001).

[5] I. Antoniadis, S. Dimopoulos, A. Pomarol, and M. Quiros, Nucl. Phys. B544, 503 (1999); I. Antoniadis, S. Dimopoulos, and G.R. Dvali, ibid. B516, 70 (1998).

[6] N. Arkani-Hamed, L.J. Hall, Y. Nomura, D.R. Smith, and N. Weiner, Nucl. Phys. B605, 81 (2001).

[7] A. Delgado, G. von Gersdorff, P. John, and M. Quiros, Phys. Lett. B 517, 445 (2001); R. Contino and L. Pilo, ibid. 523, 347 (2001); A. Masiero, C.A. Scrucca, M. Serone, and L. Silvestrini, Phys. Rev. Lett. 87, 251601 (2001).

[8] D.M. Ghilencea, S. Groot Nibbelink, and H.P. Nilles, Nucl. Phys. B619, 385 (2001).

[9] C.A. Scrucca, M. Serone, L. Silvestrini, and F. Zwirner, Phys. Lett. B 525, 169 (2002).

[10] L.J. Dixon, J.A. Harvey, C. Vafa, and E. Witten, Nucl. Phys. B261, 678 (1985); Nucl. Phys. B274, 285 (1986).

[11] C. Kounnas and M. Porrati, Nucl. Phys. B310, 355 (1988); S. Ferrara, C. Kounnas, and M. Porrati, Phys. Lett. B 206, 25 (1988); S. Ferrara, C. Kounnas, M. Porrati, and F. Zwirner, Nucl. Phys. B318, 75 (1989); C. Kounnas and B. Rostand, ibid. B341, 641 (1990).

[12] E. Kiritsis and C. Kounnas, Nucl. Phys. B503, 117 (1997); I. Antoniadis, G. D'Appollonio, E. Dudas, and A. Sagnotti, ibid.
B553, 133 (1999); C.A. Scrucca and M. Serone, J. High Energy Phys. 10, 017 (2001).

[13] M. Dine, N. Seiberg, and E. Witten, Nucl. Phys. B289, 589 (1987).

[14] M.B. Green and J.H. Schwarz, Phys. Lett. 149B, 117 (1984).

[15] N. Arkani-Hamed, A.G. Cohen, and H. Georgi, Phys. Lett. B 516, 395 (2001).

[16] P. Fayet and J. Iliopoulos, Phys. Lett. 51B, 461 (1974).

[17] R. Barbieri, S. Ferrara, D.V. Nanopoulos, and K.S. Stelle, Phys. Lett. 113B, 219 (1982).

[18] S. Ferrara, L. Girardello, T. Kugo, and A. Van Proeyen, Nucl. Phys. B223, 191 (1983).

[19] R. Barbieri, L.J. Hall, and Y. Nomura, Nucl. Phys. B624, 63 (2002).

[20] A.N. Redlich, Phys. Rev. Lett. 52, 18 (1984); Phys. Rev. D 29, 2366 (1984).

[21] L. Alvarez-Gaumé and E. Witten, Nucl. Phys. B234, 269 (1984).

[22] H. Georgi, A.K. Grant, and G. Hailu, Phys. Rev. D 63, 064027 (2001).

[23] E. Cremmer, S. Ferrara, L. Girardello, and A. Van Proeyen, Nucl. Phys. B212, 413 (1983).

[24] N. Arkani-Hamed, T. Gregoire, and J. Wacker, J. High Energy Phys. 03, 055 (2002).

[25] A. Hebecker, hep-ph/0112230.

[26] N. Arkani-Hamed, A.G. Cohen, and H. Georgi, Phys. Rev. Lett. 86, 4757 (2001); C.T. Hill, S. Pokorski, and J. Wang, Phys. Rev. D 64, 105005 (2001).

[27] E.A. Mirabelli and M.E. Peskin, Phys. Rev. D 58, 065002 (1998).

[28] A. Lukas, B.A. Ovrut, K.S. Stelle, and D. Waldram, Nucl. Phys. B552, 246 (1999).

[29] M. Gunaydin, G. Sierra, and P.K. Townsend, Nucl. Phys. B242, 244 (1984); Phys. Lett. 133B, 72 (1983).

[30] A. Ceresole and G. Dall'Agata, Nucl. Phys. B585, 143 (2000). 
[31] C.K. Zachos, Phys. Lett. 76B, 329 (1978).

[32] E. Bergshoeff, R. Kallosh, and A. Van Proeyen, J. High Energy Phys. 10, 033 (2000)

[33] R. Barbieri, L.J. Hall, and Y. Nomura, hep-ph/0110102.
[34] L. Pilo and A. Riotto, hep-th/0202144.

[35] K. Fujikawa, Phys. Rev. Lett. 42, 1195 (1979).

[36] I. Antoniadis, Phys. Lett. B 246, 377 (1990); N. ArkaniHamed, S. Dimopoulos, and G.R. Dvali, ibid. 429, 263 (1998). 